\title{
COVID-19 spreading in financial networks: A semiparametric matrix regression model
}

\author{
Monica Billio ${ }^{a, *}$, Roberto Casarin ${ }^{a}$, Michele Costola ${ }^{a}$, Matteo Iacopini ${ }^{b, c}$ \\ a Ca' Foscari University of Venice, Fondamenta San Giobbe, 873, Venice 30121, Italy \\ ${ }^{\mathrm{b}}$ Vrije Universiteit Amsterdam, De Boelelaan 1105, Amsterdam 1081 HV, The Netherlands \\ ' Tinbergen Institute, Gustav Mahlerplein 117, Amsterdam 1082 MS, The Netherlands
}

\section{A R T I C L E I N F O}

\section{Article history:}

Received 30 December 2020

Revised 10 October 2021

Accepted 10 October 2021

Available online $\mathrm{xxx}$

\section{JEL classification:}

C11

C58

G10

Keywords:

Multilayer networks

Financial markets

COVID-19

\begin{abstract}
A B S T R A C T
Network models represent a useful tool to describe the complex set of financial relationships among heterogeneous firms in the system. A new Bayesian semiparametric model for temporal multilayer networks with both intra- and inter-layer connectivity is proposed. A hierarchical mixture prior distribution is assumed to capture heterogeneity in the response of the network edges to a set of risk factors including the number of COVID-19 cases in Europe. Two layers, defined by stock returns and volatilities are considered and within and between layers connectivity is investigated. The financial connectedness arising from the interactions between two layers is measured. The model is applied in order to compare the topology of the network before and after the spreading of the COVID-19 disease.
\end{abstract}

(c) 2021 The Authors. Published by Elsevier B.V. on behalf of EcoSta Econometrics and Statistics. This is an open access article under the CC BY license (http://creativecommons.org/licenses/by/4.0/)

\section{Introduction}

The recent outbreak of the COVID-19 disease has severely affected the economy and the financial markets due to the consequences of lockdowns and travel limitations. According to the International Monetary Fund (IMF, 2020), the world growth in 2020 is projected to have a contraction of 4.4\%. During February-March of 2020, the global financial market suffered multiple crashes, with the largest drop of around $13 \%$ on March 16, 2020. To ensure financial stability and avoid the market breakdown, central banks supported the functioning of the system with asset purchase programs. While the 2007 global financial crisis originated from the vulnerabilities of the US mortgage market, which in turn was the root cause of the European sovereign debt crisis, the COVID-19 pandemic has represented an (unprecedented) exogenous shock to the financial system that cannot be reasonably foreseen, and hence, priced by the financial market. The analysis of the financial connectedness through network modeling can provide interesting insights to policymakers regarding the effect of COVID-19 on the financial system.

The literature on financial connectedness and network modeling has rapidly increased after the recent financial crisis, both theoretically (i.e., Elliott et al., 2014; Acemoglu et al., 2015) and empirically (i.e., Billio et al., 2012; Diebold and Yllmaz, 2014). Financial networks based on credit or portfolio exposures are often incomplete and only available to policy

\footnotetext{
* Corresponding author. Tel.: +390412349170.

E-mail addresses: billio@unive.it (M. Billio), r.casarin@unive.it (R. Casarin), michele.costola@unive.it (M. Costola), m.iacopini@vu.nl (M. Iacopini).
} 
authorities. Therefore, it is common practice to infer unobserved network structures by using, for instance, correlationbased or graphical approaches. The econometric methodologies proposed for extracting unobserved networks from multiple time series have been particularly flourishing (e.g., Billio et al., 2021; Skripnikov and Michailidis, 2019). Ahelegbey et al. (2016a,b) combined a Bayesian graphical approach with vector autoregressive (VAR) models, where the contemporaneous and temporal causal structures of the model are represented through two distinct graphs. Bianchi et al. (2019) proposed a Markov-switching graphical seemingly unrelated regression (SUR) model to investigate changes in systemic risk. The authors showed that the level of financial connectedness increased in 1999-2003 and 2008-2009. Using a time-varying parameter vector autoregressive model (TVP-VAR), Geraci and Gnabo (2018) estimated a dynamic Granger network in the S\&P 500 market and found a gradual decrease in network connectivity not detectable using a rolling window approach. An alternative method for network extraction was proposed using the forecast error variance decomposition (Diebold and Yilmaz, 2009; Diebold and Yilmaz, 2014), which relies on the notion of Sim's causality. Billio et al. (2019) proposed a Bayesian nonparametric Lasso prior for VAR models for a high-dimensional multivariate time-series. The causal networks were extracted through clustering and shrinking effects and well described real-world network features. Bernardi and Costola (2019) proposed a shrinkage and selection methodology for network inference through a regularized linear regression model with spike-andslab prior on the parameters. The financial linkages were expressed in terms of inclusion probabilities, which result in a weighted directed network.

The recent econometric and financial literature has focused on multilayer networks where different types of relationships between nodes are used to define multiple connectivity layers (Boccaletti et al., 2014). The use of connectivity and centrality measures that account for edges among layers can improve the understanding of the network topology. For instance, Wang et al. (2020) considered a multilayer Granger causality network inn which the layers encode different types of spillover effects: mean, volatility, and tails. The authors showed that, before a general financial turmoil, significant changes appear in the connectivity on extreme risk and volatility spillover layers. Casarin et al. (2020) proposed a Bayesian graphical vector autoregressive model to extract a multilayer network in the international oil market and showed that the oil production network is a lagged driver for prices.

Following this stream of literature, multilayer networks with both intra- and inter-layer connectivity have been considered, and the financial connectedness arising from the interactions between return and volatility layers has been measured. These connectivity effects are represented at four levels: (i) return linkages (return causes return), (ii) volatility linkages (volatility causes volatility), (iii) risk premium linkages (volatility causes return), and (iv) leverage linkages (return causes volatility).

Despite the initial research motivated by the financial crises, the literature on network data modeling in econometrics is scarce and mainly concerned with models designed for static networks (De Paula, 2017). However, when analyzing a time series of networks, such as a collection of yearly snapshots of the interbank network, dynamic models might be more adequate. Billio et al. (2018b) proposed a dynamic linear regression model for tensor-valued response variables and covariates with a parsimonious parametrization based on low-rank decomposition. In addition, Billio et al. (2018a) proposed a Bayesian Markov-switching regression model for multidimensional arrays (tensors) of binary time series. The coefficient tensor can switch between multiple regimes to capture time-varying sparsity patterns in the network structure. Outside of the econometrics literature, a few other contributions have been made to model time-varying networks, but most of them are related to the representation and description of temporally evolving graphs (e.g., Holme and Saramäki, 2012; Kostakos, 2009) or single-layer networks (e.g., Anacleto and Queen, 2017).

We contribute to the network literature by proposing a new Bayesian semiparametric model for temporal multilayer directed networks.

As network data are represented through matrices, we assume a matrix-variate distribution for the observation noise. Moreover, we assume a linear regression model for networks to investigate the role of some selected risk factors on the dynamics of the multilayer financial network. The use of matrix-valued statistical models in time-series econometrics has become increasingly popular over the last decades. In the seminal paper by Harrison and West (1999), matrix-valued distributions were exploited for representing state-space models. Recently, Carvalho and West (2007); Wang and West (2009) used the matrix normal distribution in Bayesian dynamic linear models, while Carvalho et al. (2007) applied the hyper-inverse Wishart distribution in a Gaussian dynamic graphical model. Other applications of matrix-variate distributions include stochastic volatility (Uhlig, 1997; Gouriéroux et al., 2009; Golosnoy et al., 2012; Gruber and West, 2017), classification of longitudinal datasets (Viroli, 2011), network models (Zhu et al., 2017; 2019), and factor models (Chen et al., 2019; Gao and Tsay, 2021). The novel approach makes two main contributions to the literature. First, the matrix-valued linear model is extended to panels of matrix-valued data. Second, a hierarchical mixture prior is proposed to cope with overfitting and loss of efficiency in high-dimensional settings. This prior choice allows for a semiparametric model that grants higher flexibility in investigating the impact of covariates on matrix-valued response variables. The model and inference are well suited for the analysis of multilayer temporal networks, where the intra- and inter-layer connectivity at each point in time is encoded by a cross-section of adjacency matrices.

An original application to a European financial network among 412 firms based in Germany, France, and Italy shows that the proposed framework scales well in high dimensions (i.e., hundreds of nodes) and can be successfully used to provide new insights into shock transmission in financial markets. Inspired by the literature on the causal relationship between return and volatility (i.e., Bekaert and Wu, 2000), the intra-connectivity risk premium is labeled according to the time-varying risk premium hypothesis (volatility causes returns) and the leverage is labeled according to the leverage hypothesis (return 
shocks lead to changes in volatilities). In the proposed model, the adjacency matrices of the four types of connectivity are modeled as functions of a set of risk factors, including market returns, implied volatility, corporate credit risk, and the number of COVID-19 newly confirmed European cases. In the empirical analysis, the topology of the European financial network is investigated before and after the spread of COVID-19.

The findings highlight that COVID-19 is the most relevant factor in explaining the connectivity of the European financial network at firm and sector levels, in particular industrial, real estate, and health care. The probabilities of volatility and risk premium linkages are the most positively affected by COVID-19, which also has a negative effect on leverage linkages. Moreover, we find evidence of a positive relationship between firm centrality and the number of its linkages that are impacted by COVID-19, across all layers except leverage.

The rest of the paper is structured as follows. Section 2 introduces a novel econometric framework for matrix-valued panel data, and then Section 3 presents the Bayesian inference procedure. Section 4 illustrates an empirical analysis and our major results. Finally, Section 5 concludes the study.

\section{Network Model}

Let $\mathcal{G}_{t}=\left(G_{11, t}, G_{22, t}, G_{12, t}, G_{21, t}, E\right)$ be a two-layer temporal network (Boccaletti et al., 2014), where $G_{i j, t} \subset E \times E$ is the edge set encoding the connectivity between layers $i$ and $j$, and $E=\{1, \ldots, N\}$ is the set of nodes. In the proposed framework, each node represents a firm and the two layers encode the following firm features: stock return (layer 1 ) and volatility (layer 2 ). The four graphs $G_{i j, t}, i, j=1,2$, encode the connectivity between and within layers. The connectivity is represented through the intralayer adjacency matrices $Y_{11, t}$ and $Y_{22, t}$ and the interlayer adjacency matrices $Y_{12, t}$ and $Y_{21, t}$. Here, we focus on a causal financial network in which the edges are directed and, hence, the adjacency matrices are asymmetric. Each element of the given matrix indicates how likely a causal relationship is between two variables.

For the intra-connectivity graphs, we label return linkages the sub-network $G_{11, t}$ (return causes return) and volatility linkages the sub-network $G_{22, t}$ (volatility causes volatility). Regarding the inter-connectivity graphs, we label the two graphs inspired by the causality definition in asset pricing between return and volatility as discussed by Bekaert and Wu (2000). The label risk premium linkages for the sub-network $G_{11, t}$ refers to the time-varying risk premium hypothesis (volatility causes return), while the label leverage linkages for the sub-network $G_{22, t}$ originates from the leverage hypothesis (return causes volatility).

We propose the following matrix-variate linear model for studying the impact of a set of $R$ covariates $\left(f_{1, t}, \ldots, f_{R, t}\right)$ on the linkages:

$$
\begin{aligned}
& Y_{11, t}=\sum_{r=1}^{R} B_{11, r} f_{r, t}+E_{11, t}, \quad E_{11, t} \stackrel{i i d}{\sim} \mathcal{M N}_{n, n}\left(0, \Sigma_{11,1}, \Sigma_{11,2}\right), \\
& Y_{22, t}=\sum_{r=1}^{R} B_{22, r} f_{r, t}+E_{22, t}, \quad E_{22, t} \stackrel{i i d}{\sim} \mathcal{M N}_{n, n}\left(0, \Sigma_{22,1}, \Sigma_{22,2}\right) \text {, } \\
& Y_{12, t}=\sum_{r=1}^{R} B_{12, r} f_{r, t}+E_{12, t}, \quad E_{12, t} \stackrel{i i d}{\sim} \mathcal{M N}_{n, n}\left(0, \Sigma_{12,1}, \Sigma_{12,2}\right), \\
& Y_{21, t}=\sum_{r=1}^{R} B_{21, r} f_{r, t}+E_{21, t}, \quad E_{21, t} \stackrel{i i d}{\sim} \mathcal{M N} \mathcal{N}_{n, n}\left(0, \Sigma_{21,1}, \Sigma_{21,2}\right),
\end{aligned}
$$

for $t=1, \ldots, T$, where $B_{l k, r}$ are $(n \times n)$ matrices of coefficients, $E_{l k, t}$ are error terms independent across all $l, k=1,2$ and $\forall t$, and $\mathcal{M N}_{n, n}\left(0, \Sigma_{1}, \Sigma_{2}\right)$ denotes the zero-mean matrix normal distribution with two variance/covariance matrices $\Sigma_{1}$ and $\Sigma_{2}$ (see Gupta and Nagar, 1999, Ch.2, for further details). An $(n \times p)$ random matrix $X$ is distributed as a matrix normal with mean $M$ and covariance matrices $\Sigma_{1}$ and $\Sigma_{2}$ if its density function is

$$
P\left(X \mid M, \Sigma_{1}, \Sigma_{2}\right)=(2 \pi)^{-n p / 2}\left|\Sigma_{2}\right|^{-p / 2}\left|\Sigma_{1}\right|^{-n / 2} \exp \left(-\frac{1}{2} \operatorname{tr}\left(\Sigma_{2}^{-1}(X-M)^{\prime} \Sigma_{1}^{-1}(X-M)\right)\right)
$$

with $\Sigma_{1}$ and $\Sigma_{2}$ being two positive-definite matrices of sizes $(n \times n)$ and $(p \times p)$, respectively. We write $X \sim$ $\mathcal{M N}_{n, p}\left(M, \Sigma_{1}, \Sigma_{2}\right)$. In our model, we assume $\Sigma_{l k, 1}=I_{n}$ and $\Sigma_{l k, 2}=\operatorname{diag}\left(\sigma_{1, l k}^{2}, \ldots, \sigma_{n, l k}^{2}\right)$, for each $l, k=1,2$. The motivation for our assumption is the following. First, owing to the functional form of the matrix-variate normal distribution, the identification of two covariance matrices is not possible. To address this issue, we set $\Sigma_{l k, 1}$ equal to the identity matrix. Second, to obtain a more parsimonious parametrization, we assume that the correlation among edges is mainly driven by common risk factors (systematic components). Thus, we assume that the coefficient matrices are unrestricted and the covariance matrix $\Sigma_{l k, 2}$ is diagonal. 


\section{Bayesian Inference}

\subsection{Prior specification}

As regards the prior assumption on the model parameters, we choose the following independent mixture of normal distributions for the coefficients $b_{i j, l k, r}, i, j=1, \ldots, n$ (with $i \neq j$ ), $l, k=1,2$, and $r=1, \ldots, R$ :

$$
b_{i j, l k, r} \mid \mathbf{p}_{l k}, \boldsymbol{\mu}_{l k}, \boldsymbol{\gamma}_{l k}^{2} \sim p_{1, l k} \mathcal{N}\left(b_{i j, l k, r} \mid 0, \gamma_{1, l k}^{2}\right)+\sum_{m=2}^{M_{b}} p_{m, l k} \mathcal{N}\left(b_{i j, l k, r} \mid \mu_{m, l k}, \gamma_{m, l k}^{2}\right) \text {. }
$$

To solve the label-switching problem, we impose the identification constraint $\mu_{2, l k}<\mu_{3, l k}<\ldots<\mu_{M_{b}, l k}$. See FrühwirthSchnatter (2006, Ch. 3) for a discussion on the possible solutions of the label-switching problem. As regards the prior distribution for the variances $\left(\sigma_{1, l k}^{2}, \ldots, \sigma_{n, l k}^{2}\right)$ we assume the following mixture of inverse Gamma distributions:

$$
\sigma_{i, l k, r}^{2} \mid \mathbf{q}_{l k}, \boldsymbol{\alpha}_{l k}, \boldsymbol{\beta}_{l k} \sim \sum_{m=1}^{M_{\sigma}} q_{m, l k} \mathcal{I} \mathcal{G}\left(\sigma_{i, l k}^{2} \mid \alpha_{m, l k}, \beta_{m, l k}\right),
$$

and impose the identification constraint on the mean by assuming $\beta_{1, l k} /\left(\alpha_{1, l k}-1\right)<\beta_{2, l k} /\left(\alpha_{2, l k}-1\right)<\ldots<$ $\beta_{M_{\sigma}, l k} /\left(\alpha_{M_{\sigma}, l k}-1\right)$. Finally, for the hyperparameters of the mixture prior distribution, we assume the following Dirichlet prior and normal-inverse Gamma prior distributions:

$$
\begin{aligned}
& \left(p_{1, l k}, p_{2, l k}, \ldots, p_{M_{b}, l k}\right) \sim \operatorname{Dir}\left(\phi_{b}, \phi_{b}, \ldots, \phi_{b}\right), \\
& \left(q_{1, l k}, q_{2, l k}, \ldots, q_{M_{\sigma}, l k}\right) \sim \operatorname{Dir}\left(\phi_{\sigma}, \phi_{\sigma}, \ldots, \phi_{\sigma}\right), \\
& \mu_{1, l k}=0, \\
& \gamma_{1, l k}^{2} \sim \mathcal{G} a\left(a_{0}, b_{0}\right), \\
& \mu_{m, l k} \sim \mathcal{N}\left(0, s^{2}\right), \quad m=2, \ldots, M_{b}, \\
& \gamma_{m, l k}^{2} \sim \mathcal{I} \mathcal{G}\left(a_{1}, b_{1}\right), \quad m=2, \ldots, M_{b}, \\
& \alpha_{m, l k} \sim \mathcal{G} a\left(a_{2}, b_{2}\right), \quad m=1, \ldots, M_{\sigma}, \\
& \beta_{m, l k} \sim \mathcal{G} a\left(a_{3}, b_{3}\right), \quad m=1, \ldots, M_{\sigma},
\end{aligned}
$$

which is a standard choice in Bayesian mixture modeling (Frühwirth-Schnatter, 2006). The first component of the mixture prior for coefficients $b_{i j, l k, r}$ has the Bayesian Lasso prior distribution as a special case, which corresponds to setting $a_{0}=1$ (Park and Casella, 2008). This prior specification strategy overcomes overparametrization and overfitting issues by clustering coefficients into groups and by shrinking the coefficients in the first group toward zero, thus improving the estimation efficiency in high dimensions. The hyperparameter values correspond to diffuse informative prior distributions for all parameters except for the variance of the first mixture component, $\gamma_{1, l k}^{2}$. We specify a tight prior for $\gamma_{1, l k}^{2}$ to allow for the interpretation of the first component as the "sparse" component. As the posterior distribution concentrates on a subset of the prior support, we conclude that the prior information is revised and the information content obtained from the data dominates the prior beliefs (see Figure C.12 in the Appendix). According to the experimental results, the posterior distribution is not sensitive to the choice of the hyper-parameter values. The proposed hierarchical mixture prior distribution naturally induces a mixture model for the matrix-valued observations. For each matrix $Y_{l k, t}, l, k=1,2$ and $t=1, \ldots, T$, by integrating out the parameters in the observation density, one obtains the following density:

$$
P\left(Y_{l k, t} \mid \boldsymbol{\mu}_{l k}, \boldsymbol{\gamma}_{l k}^{2}, \boldsymbol{\alpha}_{l k}, \boldsymbol{\beta}_{l k}\right)=\sum_{m^{\prime}=1}^{M_{\sigma}^{n}} \sum_{m=1}^{M_{b}^{n^{2}}} \tilde{p}_{m, l k} \tilde{q}_{m^{\prime}, l k} P\left(Y_{l k, t} \mid \tilde{\boldsymbol{\theta}}_{m, l k}^{b}, \tilde{\boldsymbol{\theta}}_{m^{\prime}, l k}^{\sigma}\right),
$$

where $\tilde{\boldsymbol{\theta}}_{m, l k}^{b}=\left(\tilde{\boldsymbol{\mu}}_{m, l k}, \tilde{\boldsymbol{\gamma}}_{m, l k}^{2}\right), \tilde{\boldsymbol{\theta}}_{m, l k}^{\sigma}=\left(\tilde{\boldsymbol{\alpha}}_{m^{\prime}, l k}, \tilde{\boldsymbol{\beta}}_{m^{\prime}, l k}\right)$, and

$$
\begin{aligned}
& P\left(Y_{l k, t} \mid \tilde{\boldsymbol{\theta}}_{m, l k}^{b}, \tilde{\boldsymbol{\theta}}_{m^{\prime}, l k}^{\sigma}\right)= \\
& =\iint P\left(Y_{l k, t} \mid B_{l k, 1}, \ldots, B_{l k, R}, \boldsymbol{\sigma}_{l k}^{2}\right) P\left(\boldsymbol{\sigma}_{l k}^{2} \mid \tilde{\boldsymbol{\theta}}_{m^{\prime}, l k}^{\sigma}\right) P\left(B_{l k, 1}, \ldots, B_{l k, R} \mid \tilde{\boldsymbol{\theta}}_{m, l k}^{b}\right) \mathrm{d} B_{l k, 1} \ldots \mathrm{d} B_{l k, R} \mathrm{~d} \boldsymbol{\sigma}_{l k}^{2} .
\end{aligned}
$$

See the Appendix for a proof. We summarize our Bayesian semiparametric model through the directed acyclic graph presented in Figure 1. 


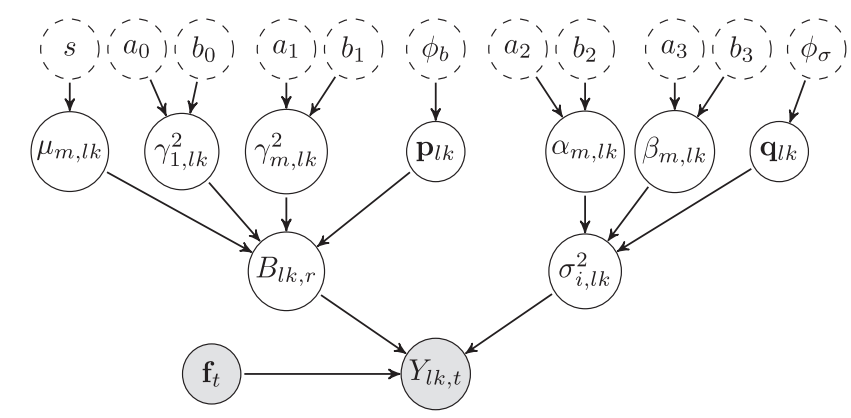

Fig. 1. Directed acyclic graph of the proposed Bayesian semiparametric model for multilayer networks. It exhibits the conditional independence structure of the observation model for $Y_{l k, t}$ with covariates $\mathbf{f}_{t}=\left(f_{1, t}, \ldots, f_{R, t}\right)^{\prime}$ (grey circles); parameters $\mathbf{p}_{l k}=\left(p_{1, l k}, \ldots, p_{M_{b}, l k}\right), \mathbf{q}_{l k}=\left(q_{1, l k}, \ldots, q_{M_{\sigma}, l k}\right), B_{l k, r}, \sigma_{i, l k}^{2}$; component-specific parameters $\mu_{m, l k}, \gamma_{m, l k}^{2}, \alpha_{m, l k}, \beta_{m, l k}$ (white solid circles); and fixed hyper-parameters $s, a_{0}, b_{0}, a_{1}, b_{1}, a_{2}, b_{2}, a_{3}, b_{3}$ (white dashed circles). The directed arrows show the causal dependence structure of the model.

\subsection{Posterior approximation}

Let us denote with $\boldsymbol{\theta}=\left(B_{l k, 1}, \ldots, B_{l k, R}, \boldsymbol{\sigma}_{l k}^{2 \prime}\right)^{\prime}$ the collection of all parameters, where $\boldsymbol{\sigma}_{l k}^{2}=\left(\sigma_{1, l k}^{2}, \ldots, \sigma_{n, l k}^{2}\right)^{\prime}$, and let $\mathbf{Y}$ and f be the collection of the observed networks and risk factors, respectively. The likelihood of the model in Eq. (1) is:

$$
\begin{aligned}
P(\mathbf{Y} \mid \boldsymbol{\theta}, \mathbf{f}) & =\prod_{t=1}^{T} \prod_{l=1}^{2} \prod_{k=1}^{2}(2 \pi)^{-n^{2} / 2}\left|\operatorname{diag}\left(\boldsymbol{\sigma}_{l k}^{2}\right)\right|^{-n / 2}\left|I_{n}\right|^{n / 2} \\
& \cdot \exp \left(-\frac{1}{2} \operatorname{tr}\left(\operatorname{diag}\left(\boldsymbol{\sigma}_{l k}^{2}\right)^{-1}\left(Y_{l k, t}-\sum_{r=1}^{R} B_{l k, r} f_{r, t}\right)^{\prime} I_{n}^{-1}\left(Y_{l k, t}-\sum_{r=1}^{R} B_{l k, r} f_{r, t}\right)\right)\right), \\
& =(2 \pi)^{-2 n^{2} T} \prod_{l=1}^{2} \prod_{k=1}^{2}\left|\operatorname{diag}\left(\boldsymbol{\sigma}_{l k}^{2}\right)\right|^{-n T / 2} \\
& \cdot \exp \left(-\frac{1}{2} \operatorname{tr}\left(\sum_{l=1}^{2} \sum_{k=1}^{2} \operatorname{diag}\left(\boldsymbol{\sigma}_{l k}^{2}\right)^{-1} \sum_{t=1}^{T}\left(Y_{l k, t}-\sum_{r=1}^{R} B_{l k, r} f_{r, t}\right)^{\prime}\left(Y_{l k, t}-\sum_{r=1}^{R} B_{l k, r} f_{r, t}\right)\right)\right) .
\end{aligned}
$$

To resolve the two mixture priors for $b_{i j, l k, r}$ and $\sigma_{i, l k}^{2}$ in Eqs. (3)-(4), we exploit a data augmentation approach and introduce two collections of allocation variables, $D_{i j, l k, r}^{b}, i, j=1, \ldots, n, l, k=1,2, r=1, \ldots, R$, and $D_{i, l k}^{\sigma}$, for $i=1, \ldots, n, l, k=1,2$. Combining this with Eqs. (3)-(4) leads to the following joint prior distributions:

$$
\begin{aligned}
& \left(b_{i j, l k, r}, d_{i j, l k, r}^{b}=m\right) \mid \mathbf{p}_{l k}, \boldsymbol{\mu}_{l k}, \boldsymbol{\gamma}_{l k}^{2} \sim p_{m, l k} \mathcal{N}\left(b_{i j, l k, r} \mid \mu_{m, l k}, \gamma_{m, l k}^{2}\right), \quad m=1, \ldots, M_{b}, \\
& \left(\sigma_{i, l k, r}^{2}, d_{i, l k}^{\sigma}=m\right) \mid \mathbf{q}_{l k}, \boldsymbol{\alpha}_{l k}, \boldsymbol{\beta}_{l k} \sim q_{m, l k} \mathcal{I} \mathcal{G}\left(\sigma_{i, l k}^{2} \mid \alpha_{m, l k}, \beta_{m, l k}\right), \quad m=1, \ldots, M_{\sigma} .
\end{aligned}
$$

As the joint posterior distribution is not tractable, we apply an MCMC approach based on Gibbs sampling to approximate the posterior distribution and all posterior quantities of interest. The Gibbs sampler iterates over the following steps:

1. Draw $\left(b_{i j, l k, 1}, \ldots, b_{i j, l k, R}\right)$ from the normal distribution $P\left(b_{i j, l k, r} \mid-\right)$.

2. Draw $\sigma_{i, l k}^{2}$ from the inverse Gamma distribution $P\left(\sigma_{i, l k}^{2} \mid-\right)$.

3. Draw the allocations $\left(d_{11, l k, r}^{b}, \ldots, d_{n 1, l k, r}^{b}, d_{12, l k, r}^{b}, \ldots, d_{n 2, l k, r}^{b}, d_{1 n, l k, r}^{b}, \ldots, d_{n n, l k, r}^{b}\right)$ from the discrete distribution $P\left(d_{i j, l k, r}^{b} \mid-\right)$.

4. Draw the allocations $\left(d_{1, l k}^{\sigma}, \ldots, d_{n, l k}^{\sigma}\right)$ from the discrete distribution $P\left(d_{i, l k}^{\sigma} \mid-\right)$.

5. Draw $\left(p_{1, l k}, \ldots, p_{M_{b}, l k}\right)$ from the Dirichlet distribution $P\left(\mathbf{p}_{l k} \mid-\right)$.

6. Draw $\left(q_{1, l k}, \ldots, q_{M_{\sigma}, l k}\right)$ from the Dirichlet distribution $P\left(\mathbf{q}_{l k} \mid-\right)$.

7. Draw the hyper-parameters:

a) $\mu_{m, l k}$, for $m=2, \ldots, M_{\sigma}$, from the normal distribution $P\left(\mu_{m, l k} \mid-\right)$.

b) $\gamma_{1, l k}^{2}$ from the generalized inverse Gaussian distribution $P\left(\gamma_{1, l k}^{2} \mid-\right)$.

c) $\gamma_{m, l k}^{2}$, for $m=2, \ldots, M_{b}$, from the inverse Gamma distribution $P\left(\gamma_{m, l k}^{2} \mid-\right)$.

d) $\alpha_{m, l k}$, for $m=1, \ldots, M_{\sigma}$, from the distribution $P\left(\alpha_{m, l k} \mid-\right)$.

e) $\beta_{m, l k}$, for $m=1, \ldots, M_{\sigma}$, from the Gamma distribution $P\left(\beta_{m, l k} \mid-\right)$. 

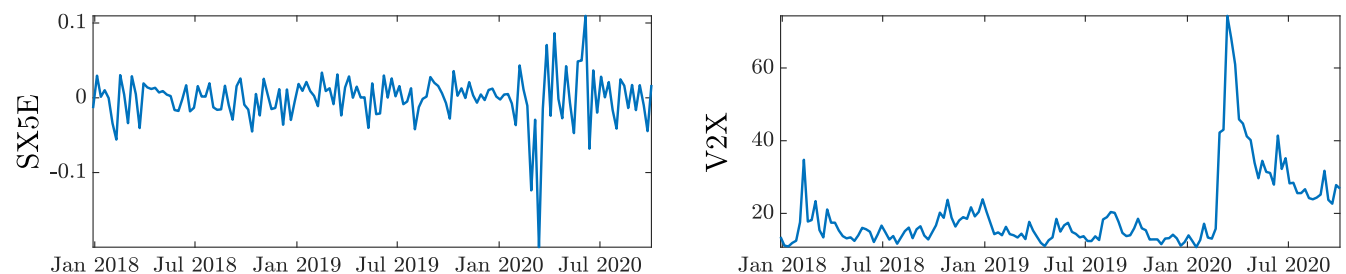

Jan 2018 Jul 2018 Jan 2019 Jul 2019 Jan 2020 Jul 2020
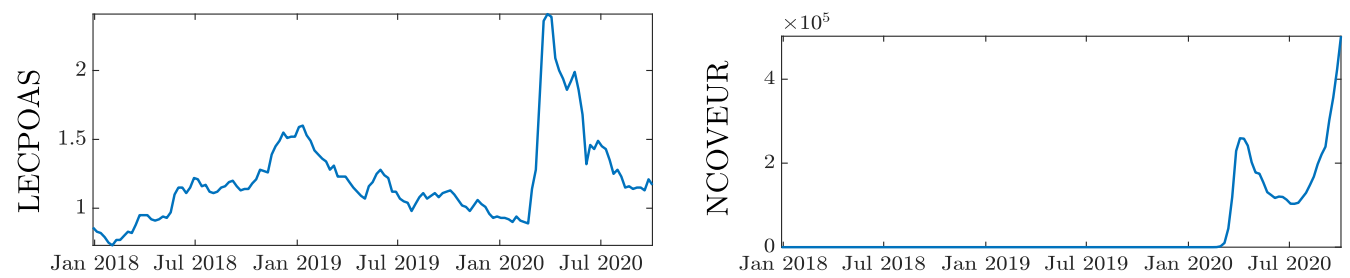

Fig. 2. The considered risk factors in the analysis: the returns on the Euro STOXX 50 index (top-left), implied volatility on the Euro STOXX 50 index (top-right), Bloomberg Barclays EuroAgg Corporate Average OAS (bottom-left), and the new European COVID-19 cases (bottom-right) for the considered European firms over time.

\section{Empirical Analysis}

In this section, we first describe the firms' dataset and the set of risk factors (source: Bloomberg and Eikon/Datastream), and then we illustrate the network extraction procedure by conducting Granger causality tests. Finally, we discuss the results obtained from the proposed network model.

\subsection{Data description}

The European firms The dataset includes 412 European firms (176 German, 162 French, and 74 Italian) belonging to 11 GICS sectors: Financials (43 firms), Communication Services (38 firms), Consumer Discretionary (61 firms), Consumer Staples (17 firms), Healthcare (48 firms), Energy (9 firms), Industrials (88 firms), Information Technology (45 firms), Materials (24 firms), Real Estate (22 firms), Utilities (12 firms), and not classified in a specific GICS sector (5 firms). The list of the firms and countries, as well as the information about their GICS sectors and industries, is available upon request from the authors. We selected Germany, France, and Italy as they are the three countries that contribute most to the European Union budget.

The data sample ranges from January 4, 2016 to September 30, 2020, at weekly frequency (Friday-Friday), thus including the period before and after the outbreak of COVID-19. The weekly logarithmic return for firm $i, r_{i, t}$, is obtained from the total returns series, whereas the weekly volatility is computed using the estimator of the variance proposed by Garman and Klass (1980):

$$
\begin{aligned}
\hat{\sigma}_{i, t}^{2}= & 0.511\left(H_{i, t}-L_{i, t}\right)^{2}-0.383\left(C_{i, t}-O_{i, t}\right)^{2} \\
& -0.019\left[\left(C_{i, t}-O_{i, t}\right)\left(H_{i, t}+L_{i, t}-2 O_{i, t}\right)-2\left(H_{i, t}-O_{i, t}\right)\left(L_{i, t}-O_{i, t}\right)\right],
\end{aligned}
$$

where $H_{i, t}$ is the weekly logarithmic high price, $L_{i, t}$ is the weekly logarithmic low price, $O_{i, t}$ is the weekly logarithmic opening price, and $C_{i, t}$ is the logarithmic closing price. The weekly prices are obtained by taking in a given week the maximum among the daily high prices (weekly High Price), the minimum among the daily low prices (weekly Low Price), the opening price of the first available day in a week (weekly Opening Price), and the closing price of the last available day in a week (weekly Closing Price).

Risk factors We consider the following common risk factors: (i) log-returns on the Euro STOXX 50 index (SX5E), (ii) implied volatility on the Euro STOXX 50 index (V2X), (iii) Bloomberg Barclays EuroAgg Corporate Average OAS (LECPOAS) as a proxy for corporate credit risk, and (iv) the new European COVID-19 cases (NCOVEUR). Figure 2 reports the plots of the factor time series for the period under investigation. In all series, there is an abrupt change during the COVID-19 outbreak.

\subsection{Network extraction}

We estimate the dynamic network of European financial institutions by using a pairwise Granger-causality test. In this respect, we follow a rolling window approach (104 observations, i.e. 2 years), which is a common practice in the financial network literature (e.g., De Nicolo and Kwast, 2002; Billio et al., 2012) and, more generally, in financial econometrics for investigating the dynamic convergence among European stock markets and portfolio optimization (Mylonidis and Kollias, 2010; Han, 2020). This method can induce temporal dependence in the estimates and can be affected by estimation bias due to the omitted variables, nevertheless it has a low computational cost. Alternative methods, such as the time-varying parameter model of Geraci and Gnabo (2018), require a substantially higher computational cost, which represents a practical 

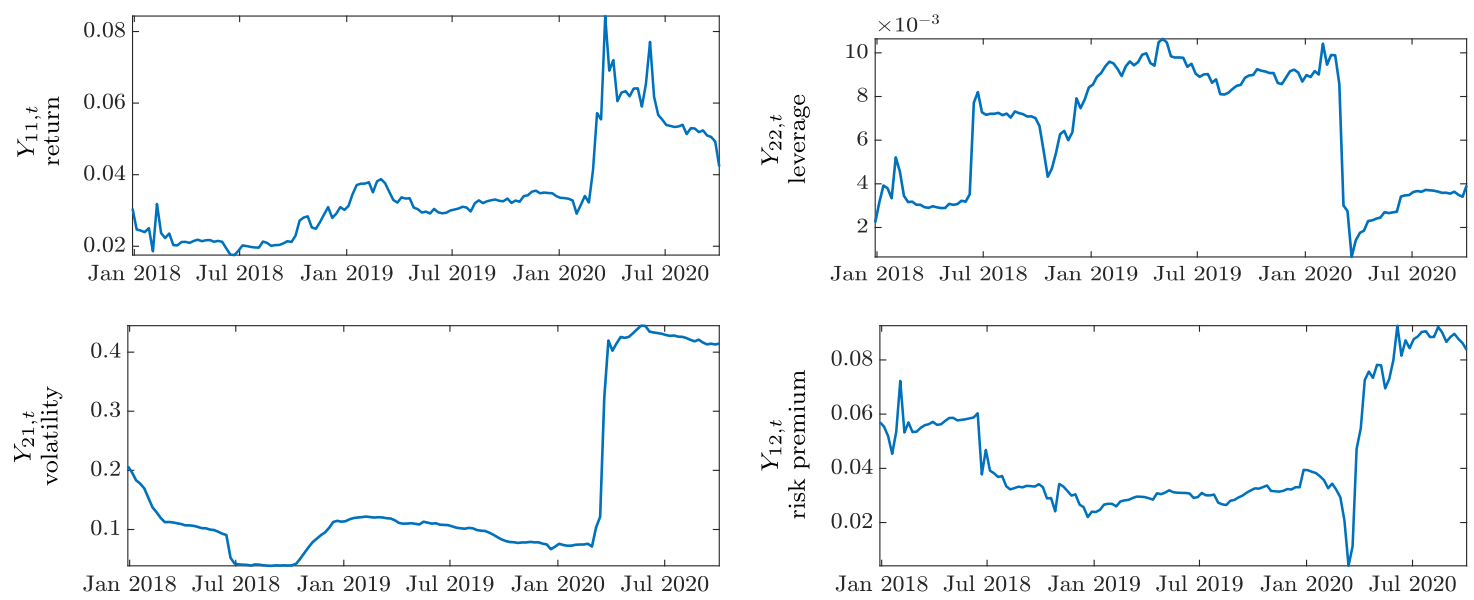

Fig. 3. Network densities (solid blue line) at $1 \%$ level of statistical significance for the return linkages (top-left), volatility linkages (bottom-left), leverage linkages (top-right), and risk premium (bottom-right) for the considered European firms over time.

concern when dealing with long time series as those in this analysis. We consider intra- and inter-connectivity, namely, return linkages, volatility linkages, risk premium linkages, and leverage linkages:

$$
\begin{aligned}
& x_{i, t}=\sum_{l=1}^{m} b_{11 l} x_{i, t-l}+\sum_{l=1}^{m} b_{12 l} x_{j, t-l}+\varepsilon_{i t}, \\
& x_{j, t}=\sum_{l=1}^{m} b_{21 l} x_{i, t-l}+\sum_{l=1}^{m} b_{22 l} x_{j, t-l}+\varepsilon_{j t},
\end{aligned}
$$

where $i, j=1, \ldots, k$ and $x_{i, t} \in\left\{r_{i, t}, \hat{\sigma}_{i, t}\right\}$. We define each entry $(i, j)$, with $i \neq j$, of the adjacency matrix $Y_{l k, t}$ associated with layer $l k$, with $l, k=2$, as the probit transformation of the p-value associated with the pairwise Granger causality test, which is $Y_{i j, l k, t}=\operatorname{probit}\left(\operatorname{pval}\left(b_{i j}\right)\right)$. Therefore, the element $Y_{i j, l k, t}$ represents a one-to-one transformation of the probability that the relationship between $x_{i, t}$ and $x_{j, t}$ is statistically significant. In our framework, as each matrix $Y_{l k, t}$ is obtained from a pairwise Granger causality test, the element $Y_{i i, l k, t}$ represents a case in which the returns (or volatility) series of institution $i$ causes itself in Granger's sense. As our focus is on the contagion dynamics (i.e., the spillover of shocks among different institutions), we follow the common practice in the financial network literature and ignore self-loops. We estimate a total of $145 \times 4$ adjacency matrices, 145 matrices for each layer, covering the period from December 29, 2017 to October 20,2020 . The estimation algorithm is parallelized and implemented in MATLAB on two nodes at the High-performance computing (HPC) cluster (VERA - Ca' Foscari University). Each node has 2 CPUs (Intel Xeon) with 20 cores (2.4 Ghz) and 768 GB of RAM.

Figure 3 presents the density of the four sub-networks over time at $1 \%$ level of statistical significance, where the network density is defined as the total number of observed linkages over the total number of possible linkages. If the density is 0 , no connection exists, while if the density is 1 , the network is fully connected. Despite sharing some similarities, the dynamics of the intra- and inter-connectivity linkages can provide different signals on shock propagation in the financial market. This calls for the joint modeling of the four connectivity layers. On average, the density is higher for the volatility linkages (0.16), followed by the risk premium linkages (0.045), return linkages (0.035), and leverage linkages (0.006). The density on the return linkages (top-left plot of Figure 3) shows two peaks on March 20, 2020, and June 5, 2020, with a reversion towards the mean after the first peak. Conversely, the density on the volatility linkages (bottom-left) shows a jump on March 27, 2020, with a new persistent higher level. Interestingly, the leverage linkages (returns cause volatility) exhibit an opposite behavior with an abrupt drop in density in March 2020. Finally, the risk premium linkages (volatility causes returns) exhibit a drop in the same month, followed by a peak in April 2020. The results of the preliminary analysis indicate that shocks on return and volatility have played different roles with heterogeneous timing during the outbreak of COVID-19 and its aftermath, thus affecting the intra- and interconnectivity differently.

The latter empirical fact can be better visualized by zooming in the period from February 21, 2020, to May 1, 2021. Accordingly, Figure 4 reports the plot of the four densities on the same scale for this sub period. The density of the returns linkages (solid line) increases until March 20, 2020, whereas that of inter-linkages decreases (dashed-dotted and dotted lines). The density of the volatility linkages (dashed line) slowly decreases until March 13, 2020, and then suddenly jumps, reaching the peak on March 27, 2020. At the same time, the density on the risk-premium linkages increases following a similar, but lagged, pattern as for the volatility linkages, whereas the number of leverage linkages start to slowly increase. This indicates that shocks on returns are followed by shocks on volatility, where the latter cause a persistent change in the level of connectivity. 

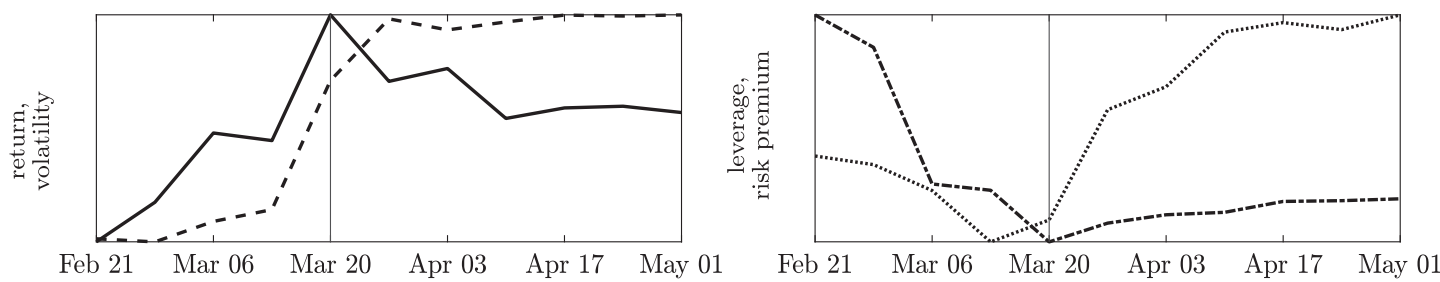

Fig. 4. Network densities at $1 \%$ level of statistical significance from February 21, 2020, to May 1, 2021 (same scale). The return linkages (solid line), volatility linkages (dashed line), leverage linkages (dashed-dotted line) and risk premium (dotted line) for the considered European firms over time. In each plot, the vertical dotted line represents March 20, 2020.

For completeness, we include in Figures 5-6 the intra- and interlayer directed networks on January 17, 2020 (one week before the first European COVID-19 case) and March 27, 2020. The connectivity increases in both the return and volatility layers. Regarding the interlayer directed networks, the connectivity of the risk premium linkages increases, while it decreases significantly in the leverage linkages. It is, therefore, interesting to apply the proposed network model to measure the impact of COVID-19 and the other risk factors on the intra- and interlinkages of the considered European firms.

\subsection{Results}

In this section, we apply the model and inference proposed in Sections 2-3 to estimate the impact of the risk factors on the multilayer European financial network. We run the MCMC algorithm for a total of 16,000 iterations. Following the results of the convergence diagnostics, we discard the first 6,000 iterations as burn-in period, and then thin down the sample 1 draw every 2 from the remaining 10,000 draws. As our analysis is focused on investigating the role of COVID-19, we describe the effects of the other risk factors (returns on the Euro STOXX 50 index, the implied volatility on the Euro STOXX 50 index, and the Bloomberg Barclays EuroAgg Corporate Average OAS) on financial linkages in the Appendix. Figure 7 reports the impact of COVID-19 on each linkage $(i, j)$, from firm $j$ to firm $i$, across all layers. For visualization purposes, only large coefficient values are reported (above 0.50 in absolute value). The blue color indicates a positive impact on the probability of an edge from firm $j$ to firm $i$, while the red color indicates a negative impact. As our response variable is an increasing transformation of the p-value of the Granger causality test performed on a pair $(i, j)$ of the time series, a negative (positive) COVID-19 coefficient implies an increase (decrease) in the p-value, and hence, in the probability to observe a linkage from $j$ to $i$. As shown in Figure 7, COVID-19 has had a mixed effect on return linkages in terms of magnitude and number of impacted linkages. Conversely, it has increased the probability of volatility and risk premium linkages, whereas, in most cases, it has reduced the probability of leverage linkages. Overall, among the selected risk factors, COVID-19 has the greatest impact on the European financial networks. The other risk factors, such as market returns, implied volatility, and corporate credit risk, have some effect on volatility and leverage linkages and a very weak effect on return and risk premium linkages (Figure C.11 in the Appendix).

The positive sign of the coefficients in the interlayer linkage equations indicates that COVID-19 has strengthened the causal relationship from volatility to returns, but not the converse. These findings support the time-varying risk premium hypothesis and show that if volatility is priced in the market, an increase in conditional volatility will require a larger return on a given stock, followed by a price reduction (e.g., see Bekaert and Wu, 2000). In our view, this follows from the increased uncertainty brought on global stock markets by COVID-19 (e.g., Engelhardt et al., 2021), which implies an increase in volatility and triggers its causal relationship with returns. In contrast, we find that COVID-19 has weakened the causal relationship from returns to volatility, meaning that there is almost no feedback effect. This suggests that the COVID-19 pandemic has enhanced a one-way spillover effect driven by a shock on the volatility rather than on returns. Therefore, it has reduced the relative explanatory power of returns in predicting the volatility.

However, the density of the leverage sub network is the lowest over the whole sample period. In particular, the density of the leverage linkages is one order of magnitude lower than that of the risk premium one (see the right column in Figure 3 ). Therefore, we hypothesisz that COVID-19 has exerted only a marginal negative impact on the returns to volatility causal relationship.

Figure 8 shows the net effect of COVID-19 on the linkages between sectors. In each panel, the block at position $(i, j)$ refers to the number of linkages (net effect) from sector $j$ to sector $i$ impacted by COVID-19. The main empirical findings are as follows:

- There is evidence of a heterogeneous net impact on return and risk premium linkages and of a substantial increase (decrease) in volatility (leverage) linkages.

- The industrial sector plays a pivotal role in the connectivity structure of the multilayer network. In return linkages, risk premium, and volatility linkages, there is an increase in the connectivity from and to the other sectors (except for consumer staples, energy, and utilities). The industrial sector exhibits the largest increase in the connectivity level within the sector (except in the leverage layer). 

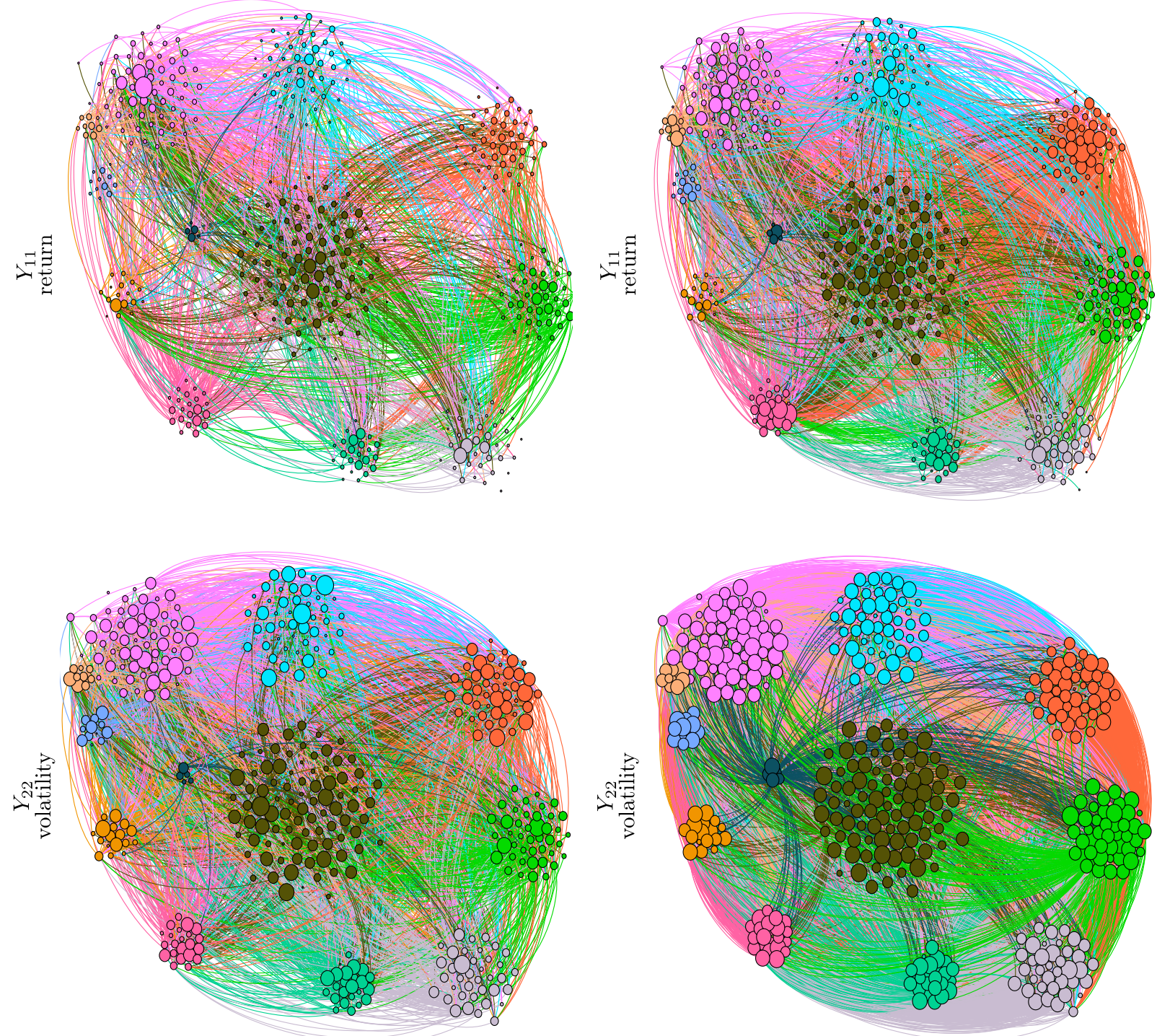

Fig. 5. The intralayer directed networks: returns linkages (top) and volatility linkages (bottom) on January 17, 2020 (left) and March 27, 2020 (right). Edges are clockwise directed. Node size: proportional to the total degree averaged over time within each regime. Edge color according to the industry of the source node: Financials (light green), Communication Services (violet), Consumer Discretionary (pink), Consumer Staples (orange), Healthcare (light blue), Energy (light orange), Industrials (olive green), Information Technology (dark orange), Materials (green), Real Estate (dark pink), Utilities (blue), and not classified in a specific GICS sector (dark green). For visualization purposes, we drop edges with weight larger than $0.1 \%$.

- In the return linkages, the financial sectors show the largest decrease in the connectivity to the other sectors (red squares in the column). Conversely, in the risk premium and volatility linkages, there is an increase in the connectivity to the other sectors (except for utilities).

- Utilities is a unique sector that exhibits a decrease in the connectivity from other sectors in the risk premium linkages, especially from the industrial sector. Similar behavior can be found also in the return linkages for the financial, real estate, and utilities sectors.

- Energy is the only sector that does not affect and is not affected by COVID-19 in all layers (except for the incoming connectivity in the return linkages).

- Healthcare and information technology are the sectors with the largest decrease in the leverage linkages from other sectors.

In conclusion, COVID-19 has impacted the European financial network between the sectors following different channels of short transmission. We further investigate the relationship between the impact of COVID-19 on financial linkages and the centrality of each firm in the network. 

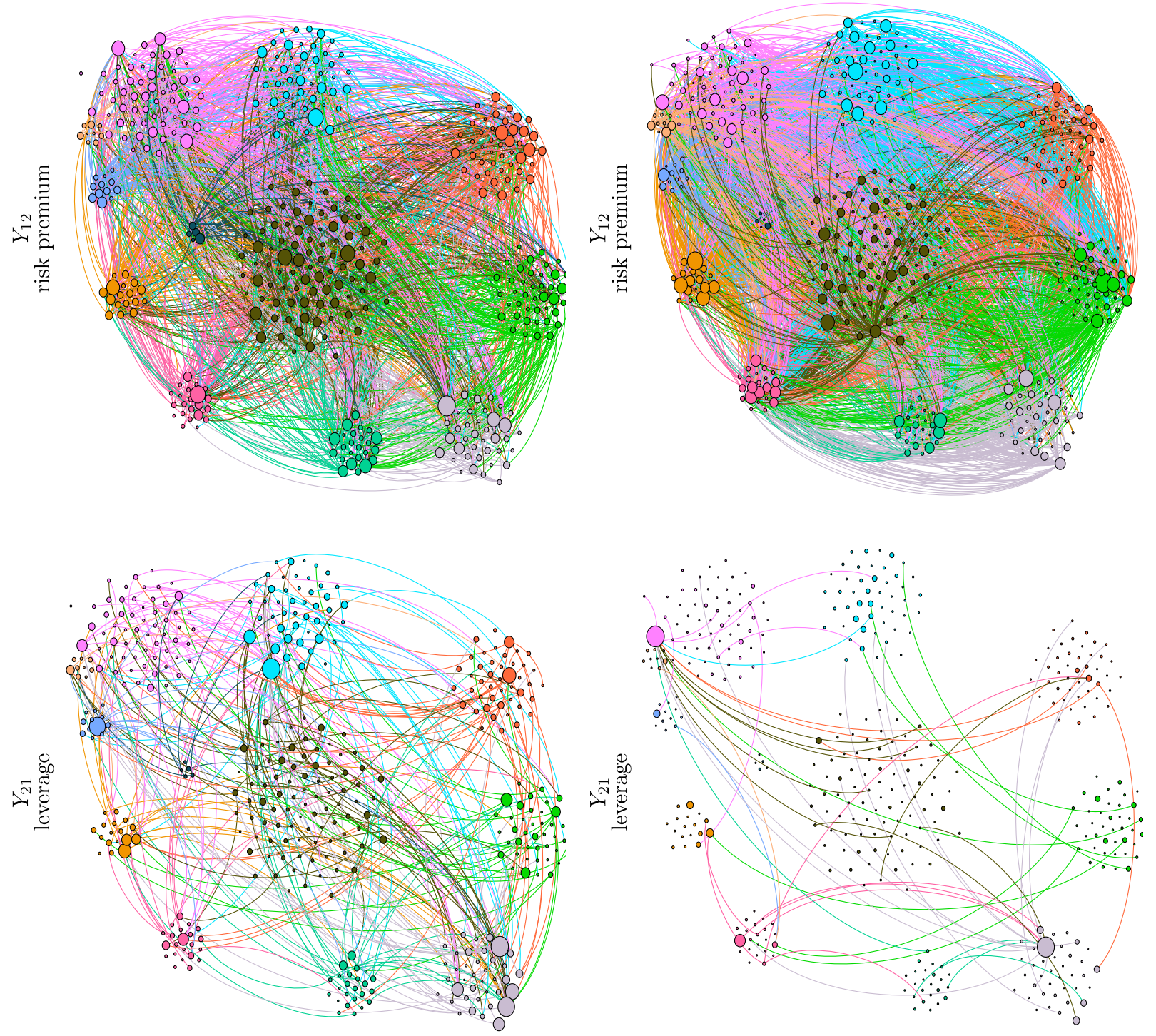

Fig. 6. The interlayer directed networks: risk premium linkages (top) and leverage linkages (bottom) on January 17, 2020 (left) and March 27, 2020 (right). Edges are clockwise directed. Node size: proportional to the total degree averaged over time within each regime. Edge color according to the industry of the source node: Financials (light green), Communication Services (violet), Consumer Discretionary (pink), Consumer Staples (orange), Healthcare (light blue), Energy (light orange), Industrials (olive green), Information Technology (dark orange), Materials (green), Real Estate (dark pink), Utilities (blue), and not classified in a specific GICS sector (dark green). For visualization purposes, we drop edges with weight larger than $0.1 \%$.

Centrality is measured either by in-/out-degree or betweenness centrality. Betweenness centrality quantifies the number of times a node acts as a bridge along the shortest path between two other nodes. Firms with large betweenness contribute to spreading contagion in the networks, thus requiring to be monitored for the stability of the financial system. For each node $i$ on layer $(l, k)$, the in-/out-degree and betweenness centrality are defined as

$$
c_{i, l k}^{I N}=\frac{1}{T} \sum_{t=1}^{T} \sum_{j=1}^{n} Y_{i j, l k, t}, \quad c_{i, l k}^{\text {OUT }}=\frac{1}{T} \sum_{t=1}^{T} \sum_{j=1}^{n} Y_{j i, l k}, \quad c_{i, l k}^{B T W}=\frac{1}{T} \sum_{t=1}^{T} \sum_{u, v \neq i} \frac{n_{u v, l k, t}(i)}{N_{u v, l k, t}},
$$

where $n_{u v, l k, t}(i)$ is the number of shortest paths from node $u$ to node $v$, on layer $(l, k)$ at time $t$, that pass through node $i$, and $N_{u v, l k, t}$ is the total number of shortest paths from $u$ to $v$, on layer $l, k$ at time $t$. We measure the impact by computing the sum of the negative (blue) and positive (red) node coefficients of a risk factor; that is,

$$
\tilde{b}_{i, l k, r}^{I N,+}=\sum_{j=1}^{n} \hat{b}_{i j, l k, r} \mathbb{I}\left(\hat{b}_{i j, l k, r}>0\right), \quad \tilde{b}_{i, l k, r}^{I N,-}=\sum_{j=1}^{n} \hat{b}_{i j, l k, r} \mathbb{I}\left(\hat{b}_{i j, l k, r}<0\right),
$$



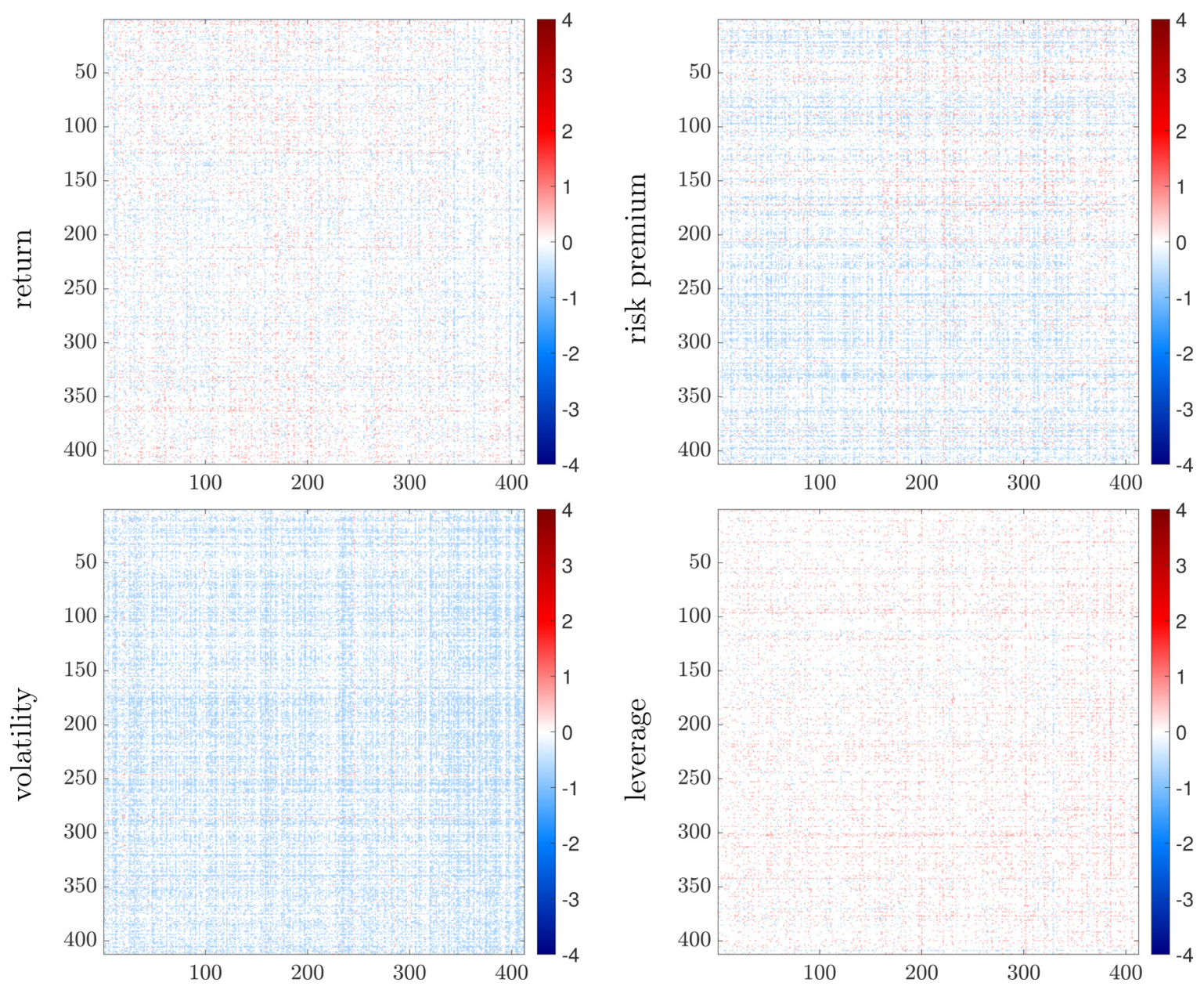

Fig. 7. Impact of COVID-19 on financial linkages for intra-layer (left column) and inter-layer (right column) networks. In each plot, the coefficient in position $(i, j)$ refers to the impact of COVID-19 on the edge from firm $j$ to firm $i$. Blue indicates positive impact on edge existence, and red indicates negative impact on edge existence. For visualization purposes, plots report coefficient estimates larger than 0.50 in absolute value.

$$
\begin{aligned}
& \tilde{b}_{i, l k, r}^{\text {OUT,+ }}=\sum_{j=1}^{n} \hat{b}_{j i, l k, r} \mathbb{I}\left(\hat{b}_{j i, l k, r}>0\right), \quad \tilde{b}_{i, l k, r}^{\text {OUT, }}=\sum_{j=1}^{n} \hat{b}_{j i, l k, r} \mathbb{I}\left(\hat{b}_{j i, l k, r}<0\right), \\
& \tilde{b}_{i, l k, r}^{B T W,+}=\sum_{j=1}^{n} \hat{b}_{i j, l k, r} \mathbb{I}\left(\hat{b}_{i j, l k, r}>0\right)+\sum_{j=1}^{n} \hat{b}_{j i, l k, r} \mathbb{I}\left(\hat{b}_{j i, l k, r}>0\right), \\
& \tilde{b}_{i, l k, r}^{B T W}=\sum_{j=1}^{n} \hat{b}_{i j, l k, r} \mathbb{I}\left(\hat{b}_{i j, l k, r}<0\right)+\sum_{j=1}^{n} \hat{b}_{j i, l k, r} \mathbb{I}\left(\hat{b}_{j i, l k, r}<0\right) .
\end{aligned}
$$

Figure 9 shows the number of linkages of each firm that are impacted by COVID-19 versus the firms' total degree. We find evidence, across the different inter- and inter-layer networks, of a positive relationship between firm centrality and the effect of COVID-19, except for leverage linkages. In particular, in the risk premium and volatility layer, almost half of the linkage of each node has been impacted by COVID-19.

Figure 10 shows the node centrality observed on March 27, 2020, versus the sum of the negative (blue) and positive (red) node coefficients. The plots in the first row report the average node in-degree, $c_{i, l k}^{I N}$, on the horizontal axis versus the sum of coefficients $\tilde{b}_{i, l k, r}^{I N,+}$ and $\tilde{b}_{i, l k, r}^{I N,-}$ on the vertical axis. The second lasts rows show similar plots for out-degree and betweenness centrality. In each plot, the triangles indicate the firms with an increased betweenness centrality after the outbreak of COVID-19. In particular, we identify the firms that moved from the 1st tercile of the betweenness centrality distribution on January 17, 2020, to the 3rd tercile on March 27, 2020.

The negative impact of COVID-19 on edge existence (red color) uniformly affects the firms with low and high centrality in the multilayer networks. This is also true for the positive impact of COVID-19 (blue color) in the leverage linkages. The 

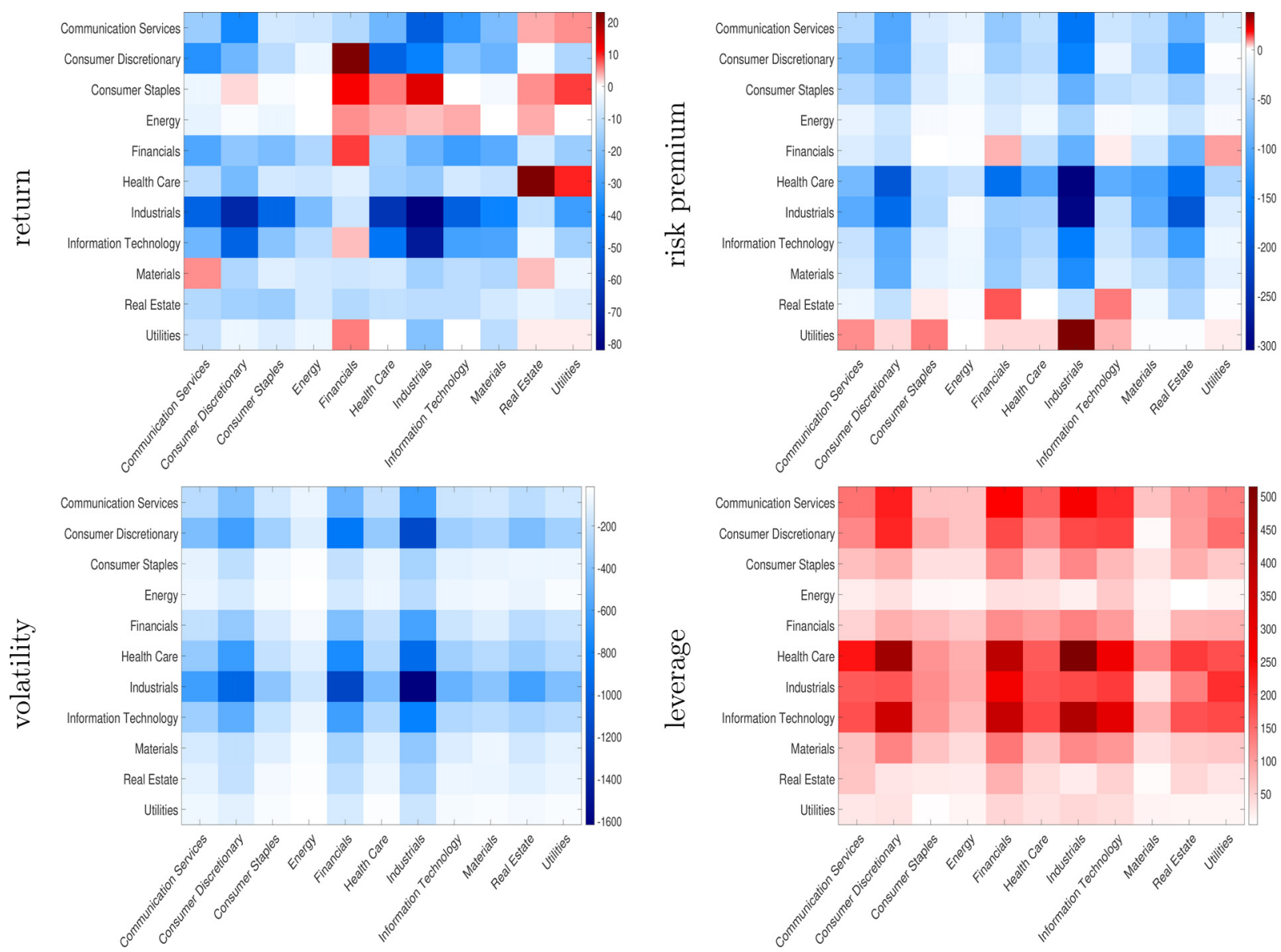

Fig. 8. Impact of COVID-19 on sector linkages for intra-layer (left column) and inter-layer (right column) networks. In each plot, the block in position ( $i, j$ ) refers to the number of linkages (net effect) from sector $j$ to sector $i$ impacted by COVID-19. Blue (red) indicates an increase (decrease) in the number of linkages.

return

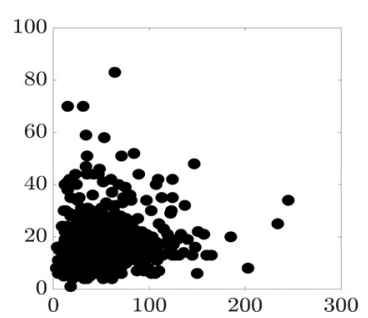

risk premium

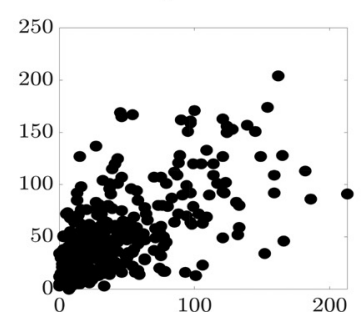

leverage

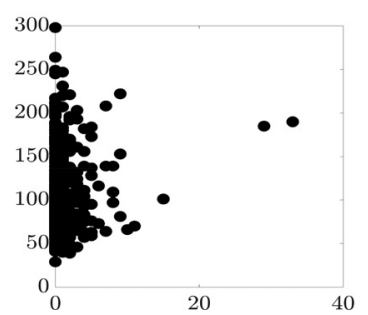

volatility

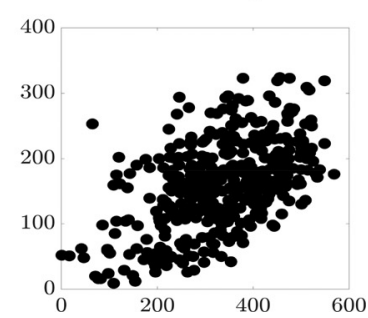

Fig. 9. Number of edges impacted by COVID-19, across layers (columns), for the nodes' total degree. In each scatterplot, node total degree on March 27, 2020 (horizontal axis) versus the number of non-null coefficients. A coefficient is considered null if its absolute value is below the threshold 0.50 .

most interesting findings concern the leverage and risk premium linkages. In the volatility linkages, there is a positive relationship between the COVID-19 coefficients and the IN degree (last column in the first row), which indicates that the connectivity of firms with higher IN degree is less affected by COVID-19. Conversely, there is a negative relationship between the COVID-19 coefficients and OUT degree (last column in the second row), which indicates that the connectivity of firms with a higher OUT degree is more affected by COVID-19. Therefore, firms with a higher OUT degree become more prone to transmitting volatility shocks to the system (last column, second row), with an impact on other firms' volatility and returns (second column). Similar conclusions can be drawn by considering the effect of COVID-19 on firms with large betweenness centrality (last column, last row). 

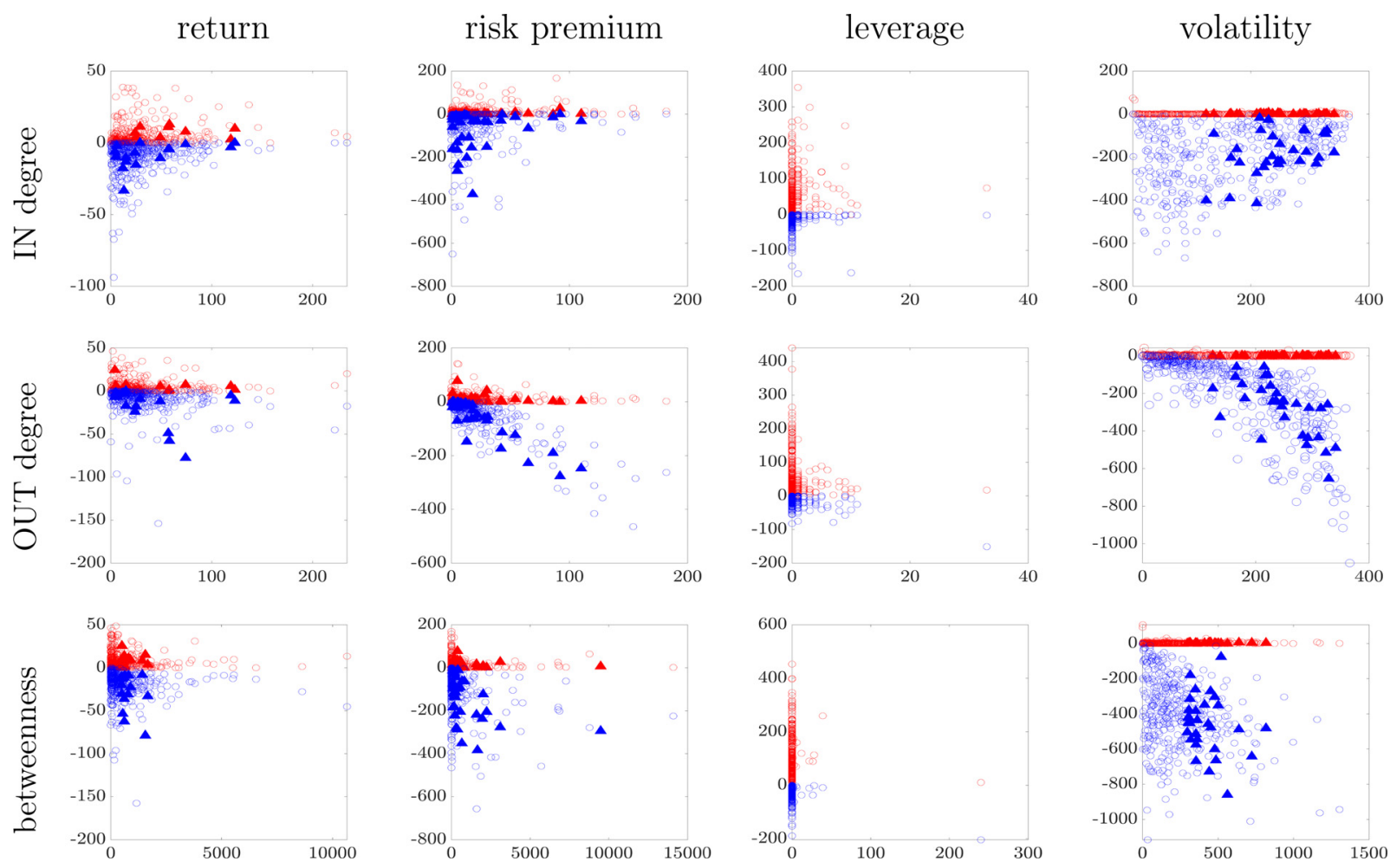

Fig. 10. Impact of the COVID-19 factors on financial linkages versus firm centrality, across layers (columns), and centrality measures (rows). In each scatterplot, node centrality on March 27, 2020 (horizontal axis) versus the sum of the negative (blue) and positive (red) node coefficients of a given variable (vertical axis). Filled triangles indicate firms that moved from the 1st tercile of the betweenness centrality distribution on January 17, 2020, to the 3rd tercile on March 27, 2020. Blue indicates positive impact on edge existence, and red indicates negative impact on edge existence. A coefficient is considered null if its absolute value is below the threshold 0.50 .

\section{Conclusion}

This paper proposes a novel Bayesian semiparametric framework for matrix-valued panel data. The model is applied to study multilayer temporal networks among European financial firms in France, Germany, and Italy. We measure the financial connectedness arising from the interactions between the two layers defined by asset returns and volatilities. The connectivity effects are represented at four levels: (i) return linkages, (ii) volatility linkages, (iii) risk premium linkages, and (iv) leverage linkages.

We have investigate the impact of COVID-19 on the network structure, which represents an unprecedented case as no previous disease outbreak has affected the real economy and the financial markets as the COVID-19 pandemic. There is evidence supporting the explanatory power of COVID-19 for the connectivity of the European financial network at firm and sector levels (e.g., industrial, real estate, and healthcare). COVID-19 has had a heterogeneous effect across layers, increasing the probabilities of volatility and risk premium linkages while decreasing the probability of leverage linkages. Finally, our results show a positive relationship between firm centrality and the number of its linkages that have been impacted by COVID-19.

Statistical modeling of financial networks can be a useful tool for policymakers and other authorities aiming at monitoring the financial system. Moreover, despite being motivated by and applied to a European financial network, the proposed econometric framework is general and can be of interest for studying a wide spectrum of matrix-variate datasets emerging in several fields of data science.

\section{Acknowledgment}

We are grateful to the Editor and two anonymous reviewers for the comments that helped improving the earlier draft of the manuscript. We thank the participants at the 14th International Conference Computational and Financial Econometrics (CFE 2020) for the useful comments. We also thank Maurizio La Mastra for the excellent research assistance. This research used SCSCF and the HPC multiprocessor cluster systems provided by the Venice Centre for Risk Analytics (VERA) at University Ca' Foscari of Venice. Monica Billio, Roberto Casarin, and Michele Costola acknowledge financial support from the Italian Ministry MIUR under the PRIN project Hi-Di NET - Econometric Analysis of High Dimensional Models with Network Structures in Macroeconomics and Finance (grant agreement no. 2017TA7TYC) and from the Venice Centre for Risk Analytics 
(VERA) at the University Ca' Foscari of Venice. Matteo Iacopini acknowledges financial support from the Marie SkłodowskaCurie Actions, European Union, Seventh Framework Program HORIZON 2020 under REA grant agreement n. 887220.

\section{Appendix A. Proof of the results in the paper}

A1. Model properties

\section{Proof of the result in Eq 13.}

$$
\begin{aligned}
P\left(Y_{l k, t} \mid \mathbf{f}_{t}, \boldsymbol{\mu}_{l k}, \boldsymbol{\gamma}_{l k}^{2}, \boldsymbol{\alpha}_{l k}, \boldsymbol{\beta}_{l k}\right)= & \iint P\left(Y_{l k, t} \mid \mathbf{f}_{t}, B_{l k, 1}, \ldots, B_{l k, R}, \boldsymbol{\sigma}_{l k}^{2}\right) P\left(\boldsymbol{\sigma}_{l k}^{2} \mid \boldsymbol{\alpha}_{l k}, \boldsymbol{\beta}_{l k}, \mathbf{q}_{l k}\right) \mathrm{d} \boldsymbol{\sigma}_{l k}^{2} \\
& \cdot P\left(B_{l k, 1}, \ldots, B_{l k, R} \mid \boldsymbol{\mu}_{l k}, \boldsymbol{\gamma}_{l k}^{2}, \mathbf{p}_{l k}\right) \mathrm{d} B_{l k, 1} \ldots \mathrm{d} B_{l k, R}, \\
= & \iint P\left(Y_{l k, t} \mid \mathbf{f}_{t}, B_{l k, 1}, \ldots, B_{l k, R}, \boldsymbol{\sigma}_{l k}^{2}\right) \sum_{m^{\prime}=1}^{M_{\sigma}^{n}} \tilde{q}_{m^{\prime}, l k} P\left(\boldsymbol{\sigma}_{l k}^{2} \mid \tilde{\boldsymbol{\alpha}}_{m^{\prime}, l k}, \tilde{\boldsymbol{\beta}}_{m^{\prime}, l k}\right) \mathrm{d} \boldsymbol{\sigma}_{l k}^{2} \\
& \cdot \sum_{m=1}^{M_{b}^{n^{2}}} \tilde{p}_{m, l k} P\left(B_{l k, 1}, \ldots, B_{l k, R} \mid \tilde{\boldsymbol{\mu}}_{m, l k}, \tilde{\boldsymbol{\gamma}}_{m, l k}^{2}\right) \mathrm{d} B_{l k, 1} \ldots \mathrm{d} B_{l k, R}, \\
= & \sum_{m^{\prime}=1}^{M_{\sigma}^{n}} \sum_{m=1}^{M_{b}^{n^{2}}} \tilde{p}_{m, l k} \tilde{q}_{m^{\prime}, l k} P\left(Y_{l k, t} \mid \mathbf{f}_{t}, \tilde{\boldsymbol{\alpha}}_{m^{\prime}, l k}, \tilde{\boldsymbol{\beta}}_{m^{\prime}, l k}, \tilde{\boldsymbol{\mu}}_{m, l k}, \tilde{\boldsymbol{\gamma}}_{m, l k}^{2}\right) .
\end{aligned}
$$

Since:

$$
\begin{aligned}
P\left(\boldsymbol{\sigma}_{l k}^{2} \mid \boldsymbol{\alpha}_{l k}, \boldsymbol{\beta}_{l k}, \mathbf{q}_{l k}\right) & =\prod_{i=1}^{n} P\left(\sigma_{i, l k}^{2} \mid \boldsymbol{\alpha}_{l k}, \boldsymbol{\beta}_{l k}, \mathbf{q}_{l k}\right), \\
& =\prod_{i=1}^{n} \sum_{m=1}^{M_{\sigma}} q_{m, l k} P\left(\sigma_{i, l k}^{2} \mid \alpha_{m, l k}, \beta_{m, l k}\right), \\
& =\sum_{i_{1}=1}^{M_{\sigma}} \ldots \sum_{i_{n}=1}^{M_{\sigma}} \prod_{u=1}^{n} q_{i_{u}, l k} P\left(\sigma_{u, l k}^{2} \mid \alpha_{i_{u}, l k}, \beta_{i_{u}, l k}\right) .
\end{aligned}
$$

By relabeling the indices and following the inverse lexicographic order:

$$
u=1+\sum_{\ell=1}^{n}\left(i_{\ell}-1\right) M_{\sigma}^{\ell-1}
$$

one obtains

$$
P\left(\boldsymbol{\sigma}_{l k}^{2} \mid \boldsymbol{\alpha}_{l k}, \boldsymbol{\beta}_{l k}, \mathbf{q}_{l k}\right)=\sum_{m^{\prime}=1}^{M_{\sigma}^{n}} \tilde{q}_{m^{\prime}, l k} P\left(\boldsymbol{\sigma}_{l k}^{2} \mid \tilde{\boldsymbol{\alpha}}_{m^{\prime}, l k}, \tilde{\boldsymbol{\beta}}_{m^{\prime}, l k}\right),
$$

where

$$
\begin{aligned}
P\left(\boldsymbol{\sigma}_{l k}^{2} \mid \tilde{\boldsymbol{\alpha}}_{m^{\prime}, l k}, \tilde{\boldsymbol{\beta}}_{m^{\prime}, l k}\right) & =\prod_{i=1}^{n} P\left(\sigma_{i, l k}^{2} \mid \alpha_{\pi\left(i, m^{\prime}\right), l k}, \beta_{\pi\left(i, m^{\prime}\right), l k}\right), \\
\tilde{q}_{m^{\prime}, l k} & =\prod_{i=1}^{n} q_{\pi\left(i, m^{\prime}\right), l k},
\end{aligned}
$$

with $\pi$ mapping the pair of indices $\left(i, m^{\prime}\right) \mapsto m$, and $m \in\left[1, M_{\sigma}\right]$. A similar argument applies to $P\left(B_{l k, 1}, \ldots, B_{l k, R} \mid \boldsymbol{\mu}_{l k}, \boldsymbol{\gamma}_{l k}^{2}, \mathbf{p}_{l k}\right)$.

\section{A2. Posterior distribution}

In the following, we provide the derivation of the full conditional distributions used in the Gibbs sampler.

The combination of the likelihood of the model in Eq. (1) and the mixture priors in Eqs. (3)-(4) yields an intractable high-dimensional integral. To address this issue, we follow the data-augmentation principle and introduce two sets of latent allocation variables, $\mathbf{D}_{l k, r}^{b}=\left\{d_{i j, l k, r}^{b}\right.$ for $\left.i, j=1, \ldots, n\right\}$, for $l, k=1,2, r=1, \ldots, R$, and $\mathbf{D}_{l k}^{\sigma}=\left\{d_{i, l k}^{\sigma}\right.$ for $\left.i=1, \ldots, n\right\}$, for $l, k=1,2$. Denote with $\boldsymbol{\alpha}_{l k}=\left(\alpha_{1, l k}, \ldots, \alpha_{M_{\sigma}, l k}\right)^{\prime}, \boldsymbol{\beta}_{l k}=\left(\beta_{1, l k}, \ldots, \beta_{M_{\sigma}, l k}\right)^{\prime}, \boldsymbol{\mu}_{l k}=\left(\mu_{1, l k}, \ldots, \mu_{M_{b}, l k}\right)^{\prime}, \gamma_{l k}^{2}=\left(\gamma_{1, l k}^{2}, \ldots, \gamma_{M_{b}, l k}^{2}\right)^{\prime}$. The data-augmented joint posterior distribution of the parameters, $B_{l k, r}$ and $\boldsymbol{\sigma}_{l k}^{2}$, the first stage hyper-parameters $\boldsymbol{\alpha}_{l k}, \boldsymbol{\beta}_{l k}, \boldsymbol{\mu}_{l k}$, 
and $\gamma_{l k}^{2}$, the latent allocation variables $\mathbf{D}_{l k, r}^{b}, \mathbf{D}_{l k}^{\sigma}$, and the mixing probabilities, $\mathbf{p}_{l k}$ and $\mathbf{q}_{l k}$, with $l, k=1,2$, and $r=1, \ldots, R$, is proportional to:

$$
\begin{aligned}
& \prod_{l=1}^{2} \prod_{k=1}^{2} \prod_{m=1}^{M_{\sigma}} P\left(\alpha_{m, l k}\right) P\left(\beta_{m, l k}\right) P\left(q_{m, l k}\right) \prod_{m=1}^{M_{b}} P\left(\mu_{m, l k}\right) P\left(\gamma_{m, l k}^{2}\right) P\left(p_{m, l k}\right) \\
& \cdot \prod_{l=1}^{2} \prod_{k=1}^{2} \prod_{i=1}^{n} P\left(\sigma_{i, l k}^{2} \mid \boldsymbol{\alpha}_{l k}, \boldsymbol{\beta}_{l k}, d_{i, l k}^{\sigma}\right) P\left(d_{i, l k}^{\sigma} \mid \mathbf{q}_{l k}\right) \prod_{j=1}^{n} \prod_{r=1}^{R} P\left(b_{i j, l k, r} \mid \boldsymbol{\mu}_{l k}, \boldsymbol{\gamma}_{l k}^{2}, d_{i j, l k, r}^{b}\right) P\left(d_{i j, l k, r}^{b} \mid \mathbf{p}_{l k}\right) \\
& \cdot \prod_{l=1}^{2} \prod_{k=1}^{2} \prod_{t=1}^{T} P\left(Y_{l k, t} \mid \mathbf{f}_{t}, B_{l k, 1}, \ldots, B_{l k, R}, \boldsymbol{\sigma}_{l k}^{2}\right) .
\end{aligned}
$$

Full conditional distribution of $b_{i j, l k, r}$ The full conditional distributions of the coefficients are given as follows. Let $\mathbf{y}_{i j, l k}=$ $\left\{y_{i j, l k, t}\right\}_{t=1}^{T}$ and $\mathbf{d}_{i j, l k}^{b}=\left\{d_{i j, l k, r}^{b}\right\}_{r=1}^{R}$ and. For each entry $(i, j)$, define $\epsilon_{i j, l k, r, t}=y_{i j, l k, t}-\sum_{r^{\prime} \neq r} b_{i j, l k, r^{\prime}} f_{r^{\prime}, t}$, thus obtaining

$$
\begin{aligned}
P\left(b_{i j, l k, r} \mid \mathbf{y}_{i j, l k}, \mathbf{f}, \mathbf{d}_{i j, l k}^{b}, \boldsymbol{\sigma}_{l k}^{2}, \boldsymbol{\mu}_{l k}, \boldsymbol{\gamma}_{l k}^{2}\right) & \propto \exp \left(-\frac{\left(b_{i j, l k, r}-\mu_{d_{i j, l k, r}^{b}}\right)^{2}}{2 \gamma_{d_{i j, l k, r}^{b}}^{2}}\right) \prod_{t=1}^{T} \exp \left(\frac{\left(\epsilon_{i j, l k, r, t}-b_{i j, l k, r} f_{r, t}\right)^{2}}{2 \sigma_{i, l k}^{2}}\right), \\
& \propto \mathcal{N}\left(\bar{\mu}, \bar{\gamma}^{2}\right),
\end{aligned}
$$

where

$$
\bar{\gamma}^{2}=\left(\frac{1}{\gamma_{d_{i j, l k, r}}^{2}}+\sum_{t=1}^{T} \frac{f_{r, t}^{2}}{\sigma_{i, l k}^{2}}\right)^{-1}, \quad \bar{\mu}=\bar{\gamma}^{2}\left(\frac{\sum_{t=1}^{T} f_{r, t} \epsilon_{i j, l k, r, t}}{\sigma_{i, l k}^{2}}+\frac{\mu_{d_{i j, l k, r}}}{\gamma_{d_{i j, l k, r}}^{2}}\right) .
$$

Full conditional distribution of $\sigma_{i, l k}^{2}$ Let $\mathbf{Y}_{l k}=\left\{Y_{l k, t}\right\}_{t=1}^{T}$ and $\mathbf{B}_{l k}=\left\{B_{l k, r}\right\}_{r=1}^{R}$. The full conditional distributions of the noise variances are given by

$$
\begin{aligned}
P\left(\sigma_{i, l k}^{2} \mid \mathbf{Y}_{l k}, \mathbf{f}, \mathbf{D}_{l k}^{\sigma}, \mathbf{B}_{l k}, \boldsymbol{\alpha}_{l k}, \boldsymbol{\beta}_{l k}\right) \propto\left(\sigma_{i, l k}^{2}\right)^{-\alpha_{d_{i, l k}^{\sigma}}-1} \exp \left(-\frac{\beta_{d_{i, l k}^{\sigma}}}{\sigma_{i, l k}^{2}}\right) & \\
& \cdot \prod_{t=1}^{T}\left(\sigma_{i, l k}^{2}\right)^{-n / 2} \exp \left(-\frac{1}{2} \operatorname{tr}\left(\operatorname{diag}\left(\boldsymbol{\sigma}_{l k}^{2}\right)^{-1}\left(Y_{l k}-\sum_{r=1}^{R} B_{l k, r} f_{r, t}\right)^{\prime}\left(Y_{l k}-\sum_{r=1}^{R} B_{l k, r} f_{r, t}\right)\right)\right), \\
\propto & \mathcal{I} \mathcal{G}\left(\alpha_{\tilde{d}_{i, l k}, l k}+\frac{T n}{2}, \beta_{\tilde{d}_{i, l k}, l k}+\frac{1}{2} \sum_{t=1}^{T} E_{i i, l k, t}\right),
\end{aligned}
$$

where $E_{l k, t}=\left(Y_{l k, t}-\sum_{r} B_{l k, r} f_{r, t}\right)^{\prime}\left(Y_{l k, t}-\sum_{r} B_{l k, r} f_{r, t}\right)$.

Full conditional distributions of $d_{i j, l k, r}^{b}$ and $d_{i, l k}^{\sigma}$ The full conditional distributions of the allocation variables are given by

$$
\begin{gathered}
P\left(d_{i j, l k, r}^{b}=m \mid b_{i j, l k, r}, \boldsymbol{\mu}_{l k}, \boldsymbol{\gamma}_{l k}^{2}, \mathbf{p}_{l k}\right) \propto p_{m, l k} \mathcal{N}\left(b_{i j, l k, r} \mid \mu_{m, l k}, \gamma_{m, l k}^{2}\right), \\
P\left(d_{i, l k}^{\sigma}=m \mid \sigma_{i, l k}^{2}, \boldsymbol{\alpha}_{l k}, \boldsymbol{\beta}_{l k}, \mathbf{q}_{l k}\right) \propto q_{m, l k} \mathcal{I} \mathcal{G}\left(\sigma_{i, l k}^{2} \mid \alpha_{m, l k}, \beta_{m, l k}\right) .
\end{gathered}
$$

Full conditional distributions of $p_{m, l k}$ and $q_{m, l k}$ The full conditional distributions of the mixing probabilities for each mixture are

$$
\begin{aligned}
& P\left(\mathbf{p}_{l k} \mid \mathbf{D}_{l k}^{b}\right) \propto \mathcal{D} \operatorname{ir}\left(\phi_{b}+\sum_{i, j, r} \mathbb{I}\left(d_{i j, l k, r}^{b}=1\right), \ldots, \phi_{b}+\sum_{i, j, r} \mathbb{I}\left(d_{i j, l k, r}^{b}=M_{b}\right)\right), \\
& P\left(\mathbf{q}_{l k} \mid \mathbf{D}_{l k}^{\sigma}\right) \propto \mathcal{D} \operatorname{ir}\left(\phi_{\sigma}+\sum_{i} \mathbb{I}\left(d_{i, l k}^{\sigma}=1\right), \ldots, \phi_{\sigma}+\sum_{i} \mathbb{I}\left(d_{i, l k}^{\sigma}=M_{\sigma}\right)\right) .
\end{aligned}
$$

Full conditional distribution of $\mu_{m, l k}$ For each $m=2, \ldots, M_{b}$, the posterior distributions of the component-specific means are obtained as

$$
P\left(\mu_{m, l k} \mid \mathbf{D}_{l k}^{b}, B_{l k, 1}, \ldots, B_{l k, R}, \gamma_{m, l k}^{2}\right) \propto \exp \left(-\frac{\mu_{m, l k}^{2}}{2 \underline{s}^{2}}\right) \prod_{\left\{i, j, r: d_{i j, l k, r}^{b}=m\right\}} \exp \left(-\frac{\left(b_{i j, l k, r}-\mu_{m, l k}\right)^{2}}{2 \gamma_{m, l k}^{2}}\right),
$$$$
\propto \mathcal{N}\left(\bar{\mu}, \bar{s}^{2}\right),
$$

where

$$
\bar{\mu}=\bar{s}^{2} \sum_{\left\{i, j, r: d_{i j, l, r}^{b}=m\right\}} \frac{b_{i j, l k, r}}{\gamma_{m, l k}^{2}}, \quad \bar{s}^{2}=\left(\frac{1}{\underline{s}^{2}}+\sum_{\left\{i, j, r: d_{i j, l k, r}^{b}=m\right\}} \frac{1}{\gamma_{m, l k}^{2}}\right)^{-1} .
$$


Full conditional distribution of $\gamma_{m, l k}^{2}$ For $m=1$, since $\mu_{1, l k}=0$, the posterior distributions of the component-specific variances are obtained as

$$
\begin{aligned}
P\left(\gamma_{1, l k}^{2} \mid \mathbf{D}_{l k}^{b}, B_{l k, 1}, \ldots, B_{l k, R}\right) & \propto\left(\gamma_{1, l k}^{2}\right)^{\underline{a}_{0}-1} \exp \left(-\frac{\gamma_{1, l k}^{2}}{\underline{b}_{0}}\right) \prod_{\left\{i, j, r: d_{i j, l k, r}^{b}=1\right\}}\left(\gamma_{1, l k}^{2}\right)^{-1 / 2} \exp \left(-\frac{b_{i j, l k, r}^{2}}{2 \gamma_{1, l k}^{2}}\right), \\
& \propto \operatorname{GiG}\left(\underline{a}_{0}-\frac{\sum_{i, j, r} \mathbb{I}\left(d_{i j, l k, r}^{b}=1\right)}{2}, \frac{2}{\underline{b}_{0}}, \sum_{\left\{i, j, r: d_{i j, l, r}^{b}=1\right\}} b_{i j, l k, r}^{2}\right),
\end{aligned}
$$

where $\operatorname{GiG}(p, a, b)$ denotes the generalized inverse Gaussian distribution with parameters $p \in \mathbb{R}, a>0$, and $b>0$, whose density function is

$$
p(x \mid p, a, b)=\frac{(a / b)^{p / 2}}{2 K_{p}(\sqrt{a b})} x^{p-1} \exp \left(-\frac{1}{2}(a x-b / x)\right),
$$

where $K_{p}$ is a modified Bessel function of the second kind. Instead, for each $m=2, \ldots, M_{b}$, the posterior distributions are

$$
\begin{aligned}
P\left(\gamma_{m, l k}^{2} \mid \mathbf{D}_{l k}^{b}, B_{l k, 1}, \ldots, B_{l k, R}, \mu_{m, l k}\right) \propto & \propto\left(\gamma_{m, l k}^{2}\right)^{-\underline{a}_{1}-1} \exp \left(-\frac{\underline{b}_{1}}{\gamma_{m, l k}^{2}}\right) \prod_{\left\{i, j, r: d_{i j, l k, r}^{b}=m\right\}}\left(\gamma_{m, l k}^{2}\right)^{-1 / 2} \exp \left(-\frac{\left(b_{i j, l k, r}-\mu_{m, l k}\right)^{2}}{2 \gamma_{m, l k}^{2}}\right), \\
& \propto \mathcal{I} \mathcal{G}\left(\underline{a}_{1}+\frac{\sum_{i, j, r} \mathbb{I}\left(d_{i j, l k, r}^{b}=m\right)}{2}, \underline{b}_{1}+\sum_{\left\{i, j, r: d_{i j, l k, r}^{b}=m\right\}} \frac{\left(b_{i j, l k, r}-\mu_{m, l k}\right)^{2}}{2}\right), \\
& \propto \operatorname{GiG}\left(-\underline{a}_{1}-\frac{\sum_{i, j, r} \mathbb{I}\left(d_{i j, l k, r}^{b}=m\right)}{2}, 0,2 \underline{b}_{1}+\sum_{\left\{i, j, r: d_{i j, l k, r}^{b}=m\right\}}\left(b_{i j, l k, r}-\mu_{m, l k}\right)^{2}\right) .
\end{aligned}
$$

Full conditional distribution of $\alpha_{m, l k}$ For $m=1, \ldots, M_{\sigma}$, the posterior distributions of the component-specific shapes are obtained as

$$
P\left(\alpha_{m, l k} \mid \mathbf{D}_{l k}^{\sigma}, \boldsymbol{\sigma}_{l k}^{2}, \beta_{m, l k}\right) \propto \alpha_{m, l k}^{\underline{a}_{2}-1} \exp \left(-\frac{\alpha_{m, l k}}{\underline{b}_{2}}\right)\left(\frac{\beta_{m, l k}^{\alpha_{m, l k}}}{\Gamma\left(\alpha_{m, l k}\right)}\right)^{\#\left\{i: d_{i, l}^{\sigma}=m\right\}}\left(\prod_{\left\{i: d_{i, l}^{\sigma}=m\right\}} \sigma_{i, l k}^{2}\right)^{-\alpha_{m, l k}} .
$$

We sample from this distribution by using an adaptive MH with RW proposal.

Full conditional distribution of $\beta_{m, l k}$ For $m=1, \ldots, M_{\sigma}$, the posterior distributions of the component-specific scales are given by

$$
\begin{aligned}
P\left(\beta_{m, l k} \mid \mathbf{D}_{l k}^{\sigma}, \boldsymbol{\sigma}_{l k}^{2}, \alpha_{m, l k}\right) & \propto \beta_{m, l k}^{a_{3}-1} \exp \left(-\frac{\beta_{m, l k}}{\underline{b}_{3}}\right) \prod_{\left\{i: d_{i, l k}^{\sigma}=m\right\}} \beta_{m, l k}^{\alpha_{m, l k}} \exp \left(-\frac{\beta_{m, l k}}{\sigma_{i, l k}^{2}}\right), \\
& \propto \mathcal{G} a\left(\underline{a}_{3}+\alpha_{m, l k} \cdot \#\left\{i: d_{i, l k}^{\sigma}=m\right\}, \underline{b}_{3}+\sum_{\left\{i: d_{i, l k}^{\sigma}=m\right\}} \sigma_{i, l k}^{2}\right) .
\end{aligned}
$$

where \#A denotes the cardinality of a set $A$.

\section{Appendix B. MCMC convergence results}

We have implemented some of the convergence diagnostic criteria provided in the Econometrics toolbox of LeSage (1999), including the autocorrelation function (ACF) at different lags, Geweke's test of equal mean, and Geweke's diagnostic based on the relative numerical efficiency (RNE).

The RNE is a measure of numerical accuracy that accounts for the autocorrelation among MCMC draws. Specifically, it provides an indication of the number of MCMC draws that would be required to get the same numerical accuracy obtainable from an iid sample from the posterior distribution. Therefore, values of the RNE close to 1.0 indicate convergence of the sampler. For each parameter of interest, $\beta$, the computation of the RNE relies on the spectral estimation of the variance of $\beta$. Since numerical issues may arise in making this approximation, alternative tapering of the spectral window are used, resulting in an estimate of the RNE based on $4 \%, 8 \%$, and $15 \%$ tapering of the spectral window. Geweke's test compares the mean of the first X\% of draws, $\mu_{1}$, against the mean of the last $Y \%, \mu_{2}$, and tests the null hypothesis of equal means, $H_{0}$ : $\mu_{1}=\mu_{2}$, against the alternative $H_{1}: \mu_{1} \neq \mu_{2}$. We choose the default values $X=20$ and $Y=50$, then, for each coefficient, we compute the p-value of the test. See LeSage (1999) for further details on these convergence diagnostic criteria.

In the empirical application, we run the MCMC algorithm for a total of 16,000 iterations. The first 6,000 are discarded as burn-in period, then we apply thinning and keep 1 draw every 2 from the remaining 10,000 draws. 


\section{Table B.1}

Convergence diagnostic criteria: autocorrelation function (ACF) at different lags, p-value of the Geweke test, and relative numerical efficiency (RNE). Each statistic is an average among 240 randomly selected entries of the coefficient matrices $B_{i j, r}$.

\begin{tabular}{|c|c|c|c|c|c|c|c|c|c|}
\hline \multicolumn{4}{|c|}{ average ACF } & \multicolumn{3}{|c|}{ average p-value Geweke test } & \multicolumn{3}{|c|}{ average RNE } \\
\hline $\operatorname{lag} 1$ & $\operatorname{lag} 5$ & lag 10 & $\operatorname{lag} 50$ & $4 \%$ taper & $8 \%$ taper & $15 \%$ taper & $4 \%$ taper & $8 \%$ taper & $15 \%$ taper \\
\hline 0.264 & 0.131 & 0.0893 & 0.0197 & 0.306 & 0.312 & 0.312 & 1.12 & 1.03 & 1.07 \\
\hline
\end{tabular}

The huge number of coefficients in the empirical application prevents us to store all the MCMC draws required to compute the above-mentioned statistics for each coefficient. Therefore, for every layer of the network, that is for every couple $(i, j), i, j=1,2$, we have randomly chosen 60 entries from the vectorized coefficient matrices $b_{i j}=\operatorname{vec}\left(\left[B_{i j, 1}, \ldots, B_{i j, R}\right]\right)$, resulting in a total of $60 \times 4=240$ entries. Then, for each of these 240 coefficients we have computed the convergence diagnostic criteria. To provide a compact summary of the results, Table B.1 shows the averages of the diagnostic criteria across entries. The outcome of all the tests, as reported in Table B.1, provide evidence of convergence of the MCMC algorithm.

\section{Appendix C. Additional results}
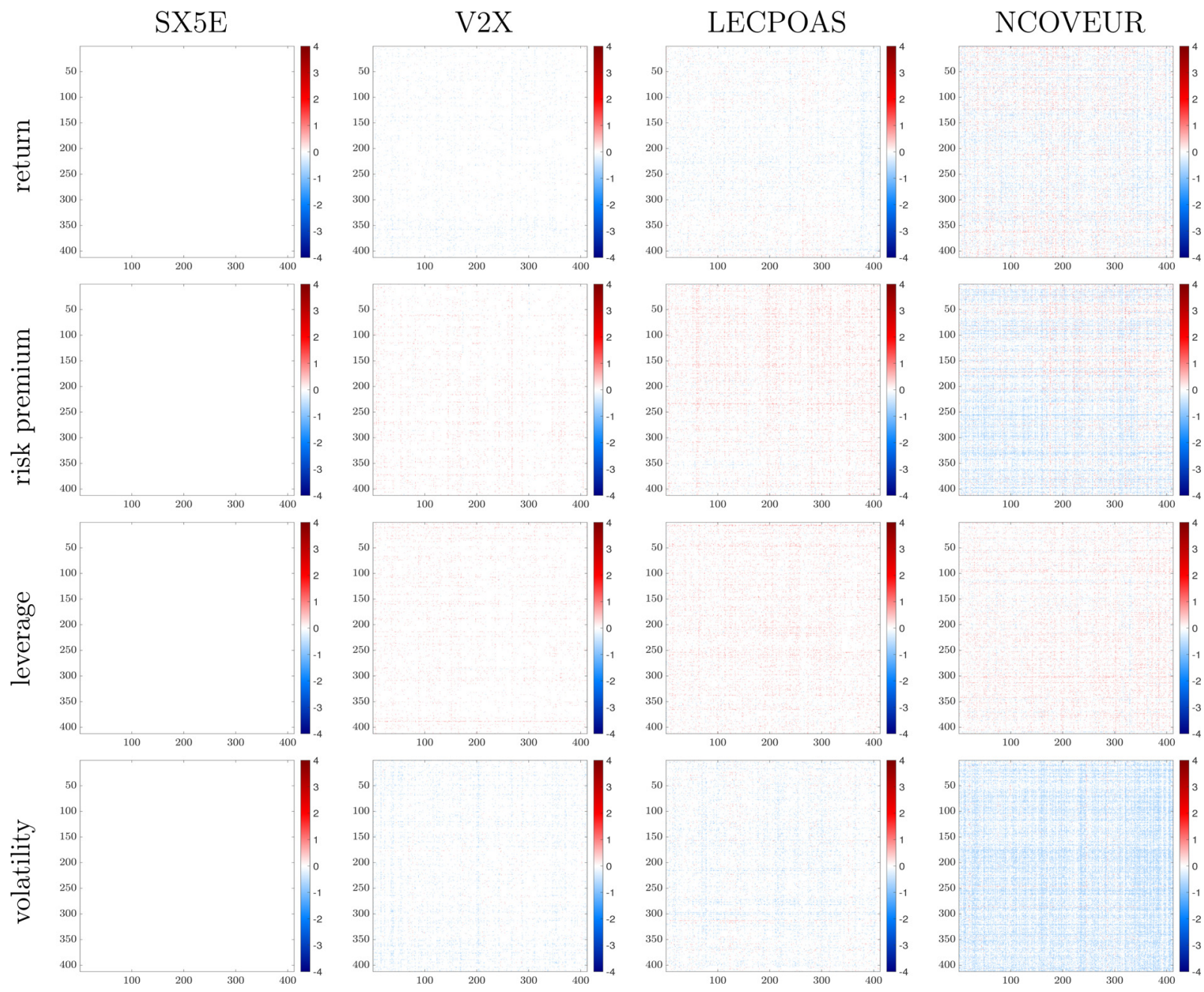

Fig. C.11. Impact of risk factors (column) on financial linkages of the multilayer network. In each plot, the coefficient in position ( $i, j)$ refers to the impact of the risk factor in column on the edge from institution $j$ to institution $i$. Blue indicates positive impact on edge existence, red indicates negative impact on edge existence. For visualization purposes, plots report coefficient estimates larger than 0.50 in absolute value. 


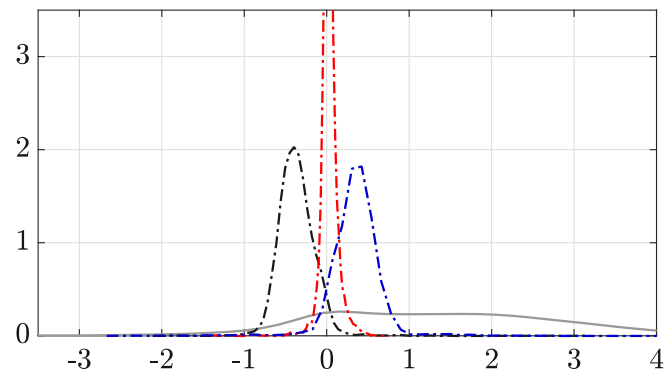

Fig. C.12. Prior distribution of the coefficients $B_{i j, l k, r}$ (solid, gray line) versus the posterior distribution of three randomly selected entries (dash-dotted lines in black, red, and blue).

\section{References}

Acemoglu, D., Ozdaglar, A., Tahbaz-Salehi, A., 2015. Systemic risk and stability in financial networks. American Economic Review 105 (2), 564-608. Ahelegbey, D.F., Billio, M., Casarin, R., 2016a. Bayesian graphical models for structural vector autoregressive processes. Journal of Applied Econometrics 31 (2), 357-386.

Ahelegbey, D.F., Billio, M., Casarin, R., 2016b. Sparse graphical vector autoregression: A Bayesian approach. Annals of Economics and Statistics (123/124) 333-361.

Anacleto, O., Queen, C., 2017. Dynamic chain graph models for time series network data. Bayesian Analysis 12 (2), $491-509$.

Bekaert, G., Wu, G., 2000. Asymmetric volatility and risk in equity markets. The Review of Financial Studies 13 (1), 1-42.

Bernardi, M., Costola, M., 2019. High-dimensional sparse financial networks through a regularised regression model. SAFE Working Paper.

Bianchi, D., Billio, M., Casarin, R., Guidolin, M., 2019. Modeling systemic risk with Markov switching graphical SUR models. Journal of Econometrics 210 (1), $58-74$.

Billio, M., Caporin, M., Frattarolo, L., Pelizzon, L., 2021. Networks in risk spillovers: A multivariate GARCH perspective. Econometrics and Statistics.

Billio, M., Casarin, R., Iacopini, M., 2018a. Bayesian Markov switching tensor regression for time-varying networks. University Ca' Foscari of Venice, Dept. of Economics WP N. 14.

Billio, M., Casarin, R., Kaufmann, S., Iacopini, M., 2018b. Bayesian dynamic tensor regression. University Ca' Foscari of Venice, Dept. of Economics WP N. 13.

Billio, M., Casarin, R., Rossini, L., 2019. Bayesian nonparametric sparse VAR models. Journal of Econometrics 212 (1), 97-115.

Billio, M., Getmansky, M., Lo, A.W., Pelizzon, L., 2012. Econometric measures of connectedness and systemic risk in the finance and insurance sectors. Journal of Financial Economics 104 (3), 535-559.

Boccaletti, S., Bianconi, G., Criado, R., Del Genio, C.I., Gómez-Gardenes, J., Romance, M., Sendina-Nadal, I., Wang, Z., Zanin, M., 2014. The structure and dynamics of multilayer networks. Physics Reports 544 (1), 1-122.

Carvalho, C.M., Massam, H., West, M., 2007. Simulation of hyper-inverse Wishart distributions in graphical models. Biometrika 94 (3), 647-659.

Carvalho, C.M., West, M., 2007. Dynamic matrix-variate graphical models. Bayesian Analysis 2 (1), 69-97.

Casarin, R., Iacopini, M., Molina, G., ter Horst, E., Espinasa, R., Sucre, C., Rigobon, R., 2020. Multilayer network analysis of oil linkages. The Econometrics Journal 23 (2), 269-296.

Chen, E.Y., Tsay, R.S., Chen, R., 2019. Constrained factor models for high-dimensional matrix-variate time series. Journal of the American Statistical Association. (forthcoming)

De Nicolo, G., Kwast, M.L., 2002. Systemic risk and financial consolidation: Are they related? Journal of Banking \& Finance 26 (5), 861-880.

De Paula, A., 2017. Econometrics of network models. In: Advances in economics and econometrics: Theory and applications, eleventh world congress. Cambridge University Press Cambridge, pp. 268-323.

Diebold, F.X., Yilmaz, K., 2009. Measuring financial asset return and volatility spillovers, with application to global equity markets. The Economic Journal 119 (534), 158-171.

Diebold, F.X., Yılmaz, K., 2014. On the network topology of variance decompositions: Measuring the connectedness of financial firms. Journal of Econometrics $182(1), 119-134$.

Elliott, M., Golub, B., Jackson, M.O., 2014. Financial networks and contagion. American Economic Review 104 (10), $3115-3153$.

Engelhardt, N., Krause, M., Neukirchen, D., Posch, P.N., 2021. Trust and stock market volatility during the COVID-19 crisis. Finance Research Letters 38 , 101873.

Frühwirth-Schnatter, S., 2006. Finite mixture and Markov switching models. Springer Science \& Business Media.

Gao, Z., Tsay, R.S., 2021. A two-way transformed factor model for matrix-variate time series. Econometrics and Statistics.

Garman, M.B., Klass, M.J., 1980. On the estimation of security price volatilities from historical data. Journal of Business 53 (1), 67-78.

Geraci, M.V., Gnabo, J.-Y., 2018. Measuring interconnectedness between financial institutions with Bayesian time-varying vector autoregressions. Journal of Financial and Quantitative Analysis 53 (3), 1371-1390.

Golosnoy, V., Gribisch, B., Liesenfeld, R., 2012. The conditional autoregressive Wishart model for multivariate stock market volatility. Journal of Econometrics 167 (1), 211-223.

Gouriéroux, C., Jasiak, J., Sufana, R., 2009. The Wishart autoregressive process of multivariate stochastic volatility. Journal of Econometrics 150 (2), 167-181.

Gruber, L.F., West, M., 2017. Bayesian online variable selection and scalable multivariate volatility forecasting in simultaneous graphical dynamic linear models. Econometrics and Statistics 3, 3-22.

Gupta, A.K., Nagar, D.K., 1999. Matrix variate distributions. CRC Press.

Han, C., 2020. A nonparametric approach to portfolio shrinkage. Journal of Banking \& Finance 120, 105953.

Harrison, J., West, M., 1999. Bayesian forecasting \& dynamic models. Springer.

Holme, P., Saramäki, J., 2012. Temporal networks. Physics Reports 519 (3), 97-125.

IMF, 2020. World Economic Outlook, October 2020: A Long and Difficult Ascent. Technical Report.

Kostakos, V., 2009. Temporal graphs. Physica A: Statistical Mechanics and its Applications 388 (6), $1007-1023$.

LeSage, J.P., 1999. Applied econometrics using MATLAB. Manuscript, Dept. of Economics, University of Toronto 154-159.

Mylonidis, N., Kollias, C., 2010. Dynamic european stock market convergence: Evidence from rolling cointegration analysis in the first euro-decade. Journal of Banking \& Finance 34 (9), 2056-2064.

Park, T., Casella, G., 2008. The Bayesian Lasso. Journal of the American Statistical Association 103 (482), 681-686.

Skripnikov, A., Michailidis, G., 2019. Joint estimation of multiple network granger causal models. Econometrics and Statistics $10,120-133$.

Uhlig, H., 1997. Bayesian vector autoregressions with stochastic volatility. Econometrica 65, 59-73.

Viroli, C., 2011. Finite mixtures of matrix normal distributions for classifying three-way data. Statistics and Computing 21 (4), $511-522$. 
Wang, G.-J., Yi, S., Xie, C., Stanley, H.E., 2020. Multilayer information spillover networks: Measuring interconnectedness of financial institutions. Quantitative Finance 1-23.

Wang, H., West, M., 2009. Bayesian analysis of matrix normal graphical models. Biometrika 96 (4), $821-834$.

Zhu, X., Pan, R., Li, G., Liu, Y., Wang, H., 2017. Network vector autoregression. The Annals of Statistics 45 (3), $1096-1123$.

Zhu, X., Wang, W., Wang, H., Härdle, W.K., 2019. Network quantile autoregression. Journal of Econometrics 212 (1), $345-358$. 